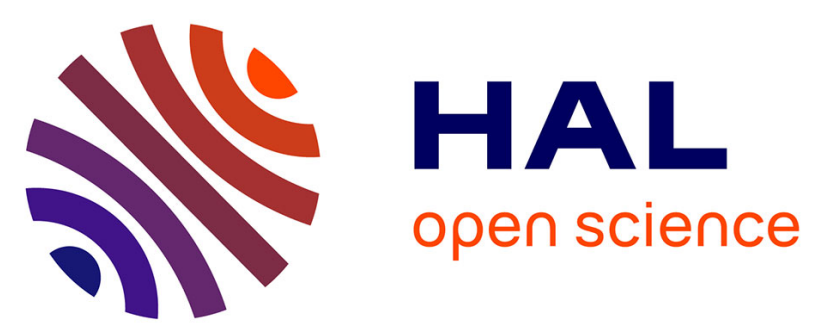

\title{
Efficient Trading Strategies
}

\author{
Elyès Jouini, Vincent Porte
}

\section{To cite this version:}

Elyès Jouini, Vincent Porte. Efficient Trading Strategies. 2007. halshs-00176616

\section{HAL Id: halshs-00176616 \\ https://shs.hal.science/halshs-00176616}

Preprint submitted on 4 Oct 2007

HAL is a multi-disciplinary open access archive for the deposit and dissemination of scientific research documents, whether they are published or not. The documents may come from teaching and research institutions in France or abroad, or from public or private research centers.
L'archive ouverte pluridisciplinaire HAL, est destinée au dépôt et à la diffusion de documents scientifiques de niveau recherche, publiés ou non, émanant des établissements d'enseignement et de recherche français ou étrangers, des laboratoires publics ou privés. 


\title{
Efficient trading strategies
}

\author{
Elyès JOUINI, \\ CEREMADE Université Paris IX-Dauphine* \\ and Institut Universitaire de France. \\ Vincent PORTE, \\ CEREMADE Université Paris IX-Dauphine and \\ G.R.O., Risk Management Group, Crédit Agricole S.A. ${ }^{\dagger}$
}

October 26, 2005

\begin{abstract}
In this paper, we point out the role of anticomonotonicity in the characterization of efficient contingent claims, and in the measure of inefficiency size of financial strategies. Two random variables are said to be anticomonotonic if they move in opposite directions. We first provide necessary and sufficient conditions for a contingent claim to be efficient in markets, which might be with frictions in a quite general framework. We then compute a measure of inefficiency size for any contingent claim. We finally give several applications of these results, studying in particular the efficiency of superreplication strategies.
\end{abstract}

Keywords: anticomonotonicity, utility maximization, markets with frictions, utility price.

\section{Introduction}

In this paper, we characterize efficient contingent claims in an economy where agents have access to a continuous securities market which might be with frictions by using the notion of anticomonotonicity. Roughly speaking, two random variables are said to be anticomonotonic if they move in opposite directions. We define an efficient consumption as an optimal choice for at least one rational agent with Von Neumann-Morgenstern preferences and a concave, strictly increasing utility function. Following the work of Jouini and Kallal [14], we compute for every contingent claim a measure of

\footnotetext{
*address: Place du Maréchal De Lattre De Tassigny - 75775 Paris Cedex 16 - France; e-mail : jouini@ceremade.dauphine.fr

${ }^{\dagger}$ address: Groupe de Recherche Opérationnelle, 91-93 Boulevard Pasteur - 75015 Paris - France; e-mail : vincent.porte@lcl.fr ; website : http://gro.creditlyonnais.fr/
} 
inefficiency that does not rely on a specific utility function, using the anticomonotonicity property. We finally provide several applications of these results.

In a couple of articles, Dybvig $[6,7]$ studied the efficiency of trading strategies, i.e. strategies chosen by at least one rational agent: given a fixed time horizon $T>0$, and a complete market with a finite number of equiprobable states of the world, agents are endowed with a concave and increasing utility function, and choose strategies maximizing their expected utility of wealth at time $T$. In this context, the no arbitrage condition implies the existence of a unique positive linear pricing rule (i.e. Arrow-Debreu price vector). A consumption bundle is then efficient if and only if it provides at least as much consumption in cheaper states of the world, that is, in states of the world with lower Arrow-Debreu prices (see Dybvig [7]). With the help of this characterization, Dybvig introduces the notion of distributional price, defined as the minimal price to obtain a given distribution; then he quantifies the inefficiency size of every contingent claim, and studies the efficiency of several trading strategies, such as the stop-loss one (see Dybvig [7]).

However, in a market with frictions, the pricing function of contingent claims is not linear anymore, but in a lot of cases, is the supremum over a set of linear pricing rules : for an incomplete market, or a market with short sales constraints or with transaction costs, Jouini and Kallal $[11,12,13]$ show that the pricing rule can be described as sublinear and obtained as the maximum over a set of underlying linear pricing rules. Jouini and Kallal [14] show that, in markets with frictions and a finite number of states of the world, the notion of distributional price is not relevant anymore. Nevertheless, they prove that the efficiency of a contingent claim is equivalent to the existence of an effective pricing vector such that the contingent claim and this pricing vector are anticomonotonic. Furthermore, they introduce the notion of "utility price", defined as the minimal price to obtain a contingent claim preferred by all rational agents, allowing them to quantify the inefficiency with a measure that does not rely on a specific utility function.

In a continuous model, if one assumes only that assets' values are semimartingales in a market with frictions, duality is more difficult to handle. In this framework, Delbaen and Schachermayer [3], Kramkov and Schachermayer [16], Hugonnier and Kramkov [10] give results of duality that we use to characterize the efficiency of strategies.

This paper is organized as follows. In a first section, we present the framework and define efficient trading strategies. In a second section, we characterize them with the help of a duality method and provide a method to compute the inefficiency size of any trading strategy (section 2). We then give applications on the computation of measure performance in markets with frictions and prove in particular that the superreplication strategy has no inefficiency size (section 3 ). 


\section{Strategies and optimization problem}

\subsection{The Economy}

We consider an economy where agents maximize their expected utility of future consumption at the final date $T>0$. Each agent is endowed with some initial wealth $x_{0}$ and some uncertain future endowment $f \leq 0$ in quantity $q_{0}$ to hedge at the final date $T$. The random variable $f$ is defined on an atomless ${ }^{1}$ probability space $(\Omega, \mathcal{F}, \mathbb{P})$.

More precisely, we use the following assumption on the preferences of the agents:

Assumption 1 Agents are characterized by a Von-Neumann Morgenstern utility function $U: \mathbb{R}_{+}^{*} \mapsto \mathbb{R}$ which is supposed to be strictly increasing and concave (not necessarily strictly concave). We denote by $\mathcal{U}$ the set of all such functions. Agents maximize their expected utility, function of their terminal wealth at $T$. We furthermore assume that they might have to hedge a contingent claim $f \leq 0$ in quantity $q_{0}>0$ at the final date $T$.

These agents have access to a securities market with frictions which consists of $d+1$ assets (one bond and $d$ stocks). We suppose that the price process $S^{0}$ of the bond is constant, i.e. $S^{0}()=$.1 , which is merely a normalization as we can always choose the bond as the numéraire, and denote by $S=\left(S^{i}\right)_{1 \leq i \leq d}$ the price process of the $d$ stocks. The process $S$ is assumed to be a semimartingale on a filtered probability space $\left(\Omega, \mathcal{F},\left(\mathcal{F}_{t}\right)_{t \in[0, T]}, \mathbb{P}\right)$, where the filtration $\left(\mathcal{F}_{t}\right)_{t \in[O, T]}$ satisfies the usual conditions with $\mathcal{F}_{T}=\mathcal{F}$.

Each agent invests its initial wealth in the securities market and builds up a self financing strategy which is defined as $(x, H)$, where $x$ is the initial value of the portfolio, $H=\left(H^{i}\right)_{1 \leq i \leq d}$ is a predictable $S$-integrable process specifying the amount of each asset held in the portfolio. The value process of this strategy is given by:

$$
V_{t} \triangleq x+(H . S)_{t}=x+\int_{0}^{t} H_{u} d S_{u}, t \in[0, T] .
$$

In order to take into account frictions on this securities market, we assume that the process $H$ has to belong to a cone $\mathcal{H}$ of the set $L^{a}(S)$, defined as the set of admissible processes $(H)_{t \in[0, T]}$, i.e. such that the stochastic integral $(H . S)$ is bounded from below. We furthermore assume that the cone $\mathcal{H}$ is closed in $L^{a}(S)$ with respect to the topology of Emery, allowing us to apply results of Föllmer and Kramkov [8]. From now on, we denote by $\mathcal{X}(x)$ the family of admissible wealth processes with initial value $x$, satisfying a

\footnotetext{
${ }^{1}$ We will use the property of atomless to construct random variables following any given distribution.
} 
no-bankruptcy condition, i.e. such that $V_{t} \geq 0$ for all $t \in[0, T]$ :

$$
\mathcal{X}(x) \triangleq\left\{(x, H) \mid x+\int_{0}^{t} H_{s} d S_{s} \geq 0, \forall t \in[0, T]\right\} .
$$

\subsection{Characteristics of the securities market}

As usual in mathematical finance, we assume that the market satisfies a condition of no arbitrage. To describe this assumption we use the concept of local supermartingale measure.

Definition 1 A probability measure $\mathbb{Q} \sim \mathbb{P}$ is called an equivalent local supermartingale measure if any (admissible) self-financing strategy $X \in \mathcal{X}(1)$ is a local supermartingale under $\mathbb{Q}$. The family of equivalent local supermartingales is denoted by $\mathcal{M}$.

In the following, we suppose that the securities market satisfies:

\section{Assumption 2}

$$
\mathcal{M} \neq \emptyset
$$

This assumption is indeed strongly related to the absence of arbitrage opportunities (see for example Delbaen and Schachermayer [3]).

We want to take into account the possibilities of frictions in the securities market. To do so, we use the cone $\mathcal{H}$ to describe the potential constraints of the market. We now review some examples of economies with frictions, specifying in each case $\mathcal{H}$.

Case 1: Market (potentially) incomplete. $\mathcal{H}=L^{a}(S)$. If the market is dynamically incomplete (without other imperfections), then the set $\mathcal{M}$ is the set of equivalent local martingales, reduced to a singleton in the case of a complete market (see e.g., Karatzas and Shreve [15]).

Case 2: Prohibition of short selling. $\mathcal{H}=\left\{H \in L^{a}(S) \mid H^{i} \geq 0,1 \leq i \leq m\right\}$. In such a market, the first $m$ assets cannot be held in negative quantities.

Case 3: Short selling costs. The introduction of shadow prices is a way to model such constraints. Assume that $d=2 m$ and $\left(S^{i}\right)_{1 \leq i \leq m}$ are the values of the assets when taken in a long position; $\left(\tilde{S}^{i}\right)_{1 \leq i \leq m} \triangleq$ $\left(S^{i}\right)_{m+1 \leq i \leq d}$ are the return processes of the same assets, but when taken in a short position, with :

$$
\tilde{S}^{i}{ }_{t} \geq S_{t}^{i}
$$

The cone $\mathcal{H}$ is then given by:

$$
\mathcal{H}=\left\{H \in L^{a}(S) \mid H^{i} \geq 0,1 \leq i \leq m \text {, and } H^{i} \leq 0, m+1 \leq i \leq d\right\} .
$$




\subsection{Efficient contingent claims}

On this quite general framework, we want to characterize efficient contingent claims (and the minimum costs of the trading strategies leading to them), defined as the optimal choice for at least one rational agent, given its uncertain future endowment to hedge. In this paragraph, we are going first to describe the set of strategies that an agent can choose. We will then define precisely the notion of efficient contingent claims.

Agents with an initial wealth $x>0$ and a quantity $q>0$ of random endowment $f$ to hedge choose their optimal trading strategy in the set

$$
\mathcal{X}(x, q) \triangleq\left\{X \in \mathcal{X}(x) \mid X_{T}+q . f \stackrel{\text { a.s. }}{\geq} 0\right\} .
$$

Note that, for some vector $(x, q), \mathcal{X}(x, q)$ may be empty, or singulary small, possibly reduced to a unique trading strategy. To exclude such situations where the problem of expected utility maximization becomes trivial, we work with the set $\mathcal{K}$ defined as the interior, in $\mathbb{R}_{+}^{2}$ of the vectors $(x, q)$ such that $\mathcal{X}(x, q)$ is not empty:

$$
\mathcal{K} \triangleq \operatorname{int}\left\{(x, q) \in \mathbb{R}_{+}^{2} \mid \mathcal{X}(x, q) \neq \emptyset\right\} .
$$

Finally, let $\mathcal{C}(x, q)$ be the set of integrable terminal wealth $c$ such that there exists a self-financing portfolio $\mathrm{X}$ with initial value $x$, i.e. $X \in \mathcal{X}(x)$, and whose terminal wealth dominates $c$ :

$\mathcal{C}(x, q) \triangleq\left\{c \geq 0, c \in L^{1} \mid\right.$ there exists $X \in \mathcal{X}(x)$ such that $\left.X_{T}+q f \stackrel{\text { a.s. }}{\geq} c\right\}$.

We shall need to consider $\operatorname{Adm}(q, f)$, defined as the set of admissible contingent claims attainable by at least one initial wealth, i.e.:

$$
\operatorname{Adm}(q, f) \triangleq \bigcup_{x \in \mathbb{R}_{+}^{*}} \mathcal{C}(x, q) .
$$

Agents choose a strategy in order to maximize their expected utility of terminal wealth:

Definition 2 Let $\left(x_{0}, q_{0}\right) \in \mathcal{K}$. A contingent claim $c_{0} \in \mathcal{C}\left(x_{0}, q_{0}\right)$ is efficient for the random endowment $f$ in quantity $q_{0}$, and the initial wealth $x_{0}$ if there exists a utility function $U \in \mathcal{U}$ such that $c_{0}$ solves

$$
\sup _{c \in \mathcal{C}\left(x_{0}, q_{0}\right)} \mathbb{E}(U(c))<+\infty .
$$

Note that we do not assume agents to be strictly risk adverse, neither need we to impose any regularity condition on utility function, as usually 
done in the literature. Indeed, papers in the literature are particulary interested in the existence of an optimal strategy maximizing the expected utility of terminal wealth (see Kramkov and Schachermayer [16], Cvitanic and al. [2], or Hugonnier and Kramkov [10]), and such assumptions are crucial to prove such an existence. However, our concern is quite different since our issue is to characterize the necessary and sufficient conditions for a strategy to be optimal for at least one utility function. Thus, it is important not to restrict the set of utility functions based on regularity conditions, as we do not want to exclude possible optimal strategies on such grounds. For the same reason, we need to restrict the set of terminal wealth to integrable random variables in order to ensure the existence of all expected utilities functions of terminal wealth, whatever the preferences of the agent.

\subsection{Description of the pricing rule}

In this paragraph, we specify the pricing rule of any contingent claim at the date $T$. In a market with frictions, the pricing rule is not linear anymore, and is defined as the supremum over a set of density pricing measures. The assumption (2) of no arbitrage, and the specifications of frictions described with the cone $\mathcal{H}$ allow us to compute the pricing rule using the results of Föllmer and Kramkov [8]. Let $c$ be a positive contingent claim at date $T$, we define $\pi(c)$ as the superreplication price of the contingent claim $c: \pi(c)$ is the minimum cost of a self-financing strategy leading to a terminal wealth almost surely bigger than $c$. One can see easily that the pricing rule $\pi$ is sublinear, i.e. $\pi\left(\lambda c_{1}\right)=\lambda \pi\left(c_{1}\right)$ and $\pi\left(c_{1}+c_{2}\right) \leq \pi\left(c_{1}\right)+\pi\left(c_{2}\right)$, for every $c_{1}$ and $c_{2}$ positive contingent claims and every nonnegative real number $\lambda \geq 0$. From a direct application of Proposition 4.2 of Föllmer and Kramkov [8], we derive the following superreplication

Theorem 1 Assume that assumption (2) holds. Then, for every positive contingent claim $c$, there exists an admissible self-financing strategy $(\pi(c), \hat{H})$ on the constrained market such that $V_{T}=\pi(c)+\int_{0}^{T} \hat{H}_{s} d S_{s} \stackrel{\text { a.s. }}{\geq} \mathrm{c}$ and:

$$
\pi(c)=\sup _{\mathbb{Q} \in \mathcal{M}} \mathbb{E}^{\mathbb{Q}}(c) .
$$

In a discrete model of market with frictions, Jouini and Kallal [14] characterize the efficiency of any contingent claim $c$ with the anticomonotonocity property with at least one effective linear pricing rule $h_{0}$, that is such that $\pi(c)=\mathbb{E}\left(h_{0} c\right)$. But in our context (with a continuous market model), there exist some contingent claims such that the supremum in the definition of $\pi$ is not attained, and duality results are more difficult to obtain. We are going first to define particular sets that we will need in order to characterize the efficiency of contingent claims, allowing us to study the case of a random endowment to hedge. 
Without random endowment, one needs to study the set $\mathcal{C}(x)$ of terminal wealth attainable from trading strategy in $\mathcal{X}(x)$ for some initial portfolio $x>0$ :

$$
\mathcal{C}(x) \triangleq\left\{c \geq 0 \mid \exists X \in \mathcal{X}(x) \text { with } X_{T} \stackrel{\text { a.s. }}{\geq} c\right\} .
$$

Following Kramkov and Schachermayer [16], this leads to consider the set of supermartingales

$$
\mathcal{Y}(y) \triangleq\left\{Y \mid Y_{0}=y \text { an } X Y \text { is a supermartingale for every } X \in \mathcal{X}(1)\right\} .
$$

and:

$$
\mathcal{D}(y) \triangleq\left\{h \mid 0 \leq h \leq Y_{T} \text { for at leat one } Y \text { belonging to } \mathcal{Y}(y)\right\} .
$$

In the case of incomplete markets, Kramkov and Schachermayer [16] prove a bipolar relationship between the sets $\mathcal{C}(x)$ and $\mathcal{D}(y)$ which is a key result to establish that there is no gap between the primal and the dual problem of the expected utility maximization problem.

If we consider the case of a random endowment to hedge, we assume first that the random variable $-f$ is effectively hedgeable:

\section{Assumption 3}

$$
\hat{x}=\sup _{\mathbb{Q} \in M} \mathbb{E}^{\mathbb{Q}}[-f]<+\infty
$$

Under this assumption, there exists an admissible strategy $(\hat{x}, \hat{\pi}, \hat{C})$ such that the terminal value of this strategy dominates $-f$ (see Theorem (1)), i.e. the set $\mathcal{C}(\hat{x})$ is not empty. Following the work of Hugonnier and Kramkov [10], one needs to consider the sets $\mathcal{X}(x, q)$ and $\mathcal{C}(x, q)$ as well as the measures of pricing $\mathcal{Y}(y, r)$ and $\mathcal{D}(y, r)$, for $y \geq 0$ and $r \leq 0$, defined as:

$$
\begin{aligned}
& \mathcal{Y}(y, r) \triangleq\left\{Y \in \mathcal{Y}(y) \mid \mathbb{E}\left(Y_{T} X_{T}\right) \leq x y+q r, \forall X_{T} \in \mathcal{X}(x, q)\right\} \\
& \mathcal{D}(y, r) \triangleq\left\{h \mid 0 \leq h \leq Y_{T} \text { for at least one } Y \in \mathcal{Y}(y, r)\right\}
\end{aligned}
$$

In the same way as for the case without random endowment, we introduce $\pi_{q f}(c)$ as the minimum cost to fund a strategy leading to a terminal wealth 
bigger than $c$, after hedging $f$ in quantity $q$. Defining $\mathcal{G}^{2}$ by:

$$
\mathcal{G} \triangleq\left\{(y, r) \in \mathbb{R}_{+} \times \mathbb{R}_{-} \mid \mathcal{D}(y, r) \neq \emptyset\right\}
$$

and denoting by $\tilde{\mathcal{P}}$ the set $\left\{p \in \mathbb{R}_{-} \mid(1, p) \in \mathcal{G}\right\}$, one can see, using duality results provided in appendix, that $\tilde{\mathcal{P}}$ is a closed convex set and that the pricing function $\pi_{q f}$ satisfies:

Theorem 2 The minimum cost $\pi_{q f}(c)$ of funding a strategy leading to a terminal wealth bigger than $c$, after hedging $f$ in quantity $q$, is given by:

$$
\pi_{q f}(c)=\sup _{p \in \tilde{\mathcal{P}}} \sup _{h \in \mathcal{D}(1, p)} \mathbb{E}(h c)-q p
$$

\section{Trading strategies efficiency}

In a complete model with a finite number of states of the world, Dybvig [6] characterizes efficient strategies with the following property: the lower the price of the Arrow-Debreu security for a state of the world, the higher the value of an efficient consumption bundle. This very intuitive result states a relationship of anticomonotonicity between the density of pricing and the efficient contingent claim. We begin this section by introducing a formal definition of the (anti)comonotonicity property. We then characterize the efficient contingent claims and finally provide a method to compute the inefficiency size of any trading strategies.

\subsection{Comonotonicity property}

Definition 3 Two real-valued random variables $c$ and $h$ defined on the same probability space $(\Omega, F, P)$ are comonotonic if one of the following equivalent conditions is satisfied

1. there exists $A$ in $\mathcal{F}$, with probability one, and such that

$$
\left[c(\omega)-c\left(\omega^{\prime}\right)\right]\left[h(\omega)-h\left(\omega^{\prime}\right)\right] \geq 0 \quad \text { for all }\left(\omega, \omega^{\prime}\right) \in A \times A
$$

\footnotetext{
${ }^{2}$ One can prove that:
}

$$
\mathcal{G}=\left\{(y, r) \in \mathbb{R}_{+} \times \mathbb{R}_{-} \mid x y+q r \geq 0 \text { for all }(x, q) \in \mathcal{K}\right\}
$$

Indeed, the inclusion $\subset$ is straightforward. For the converse inclusion, let $y \geq 0, r \leq 0$ with $x y+q r \geq 0$, for every $(x, q) \in \mathcal{K}$. Since $(x, 0)$ with $x \geq 0$ belongs to $\mathcal{K}$, necessarily $y \geq 0$, and one can find a process $Y \in \mathcal{Y}(y)$ (e.g. $Y=y\left(\frac{\bar{d} \mathbb{Q}}{d \mathbb{P}}\right)$ for some $\left.\mathbb{Q} \in \mathcal{M}\right)$. If we define the process $Z$ by:

$$
Z_{t}= \begin{cases}Y_{t}, & t<T \\ 0 & t=T\end{cases}
$$

$Z$ belongs to $\mathcal{Y}(y, r)$ and therefore $(y, r) \in \mathcal{G}$. 
2. if $\mathbb{P}^{\otimes 2}$ denotes the product probability on the space $\left(\Omega^{2}, \mathcal{F}^{\otimes 2}\right)$

$$
\mathbb{P}^{\otimes 2}\left[\left[c(\omega)-c\left(\omega^{\prime}\right)\right]\left[h(\omega)-h\left(\omega^{\prime}\right)\right] \leq 0\right]=1 .
$$

3. the cumulative distribution function $F_{(c, h)}$ of the pair $(c, h)$ is given by

$$
F_{c, h}\left(\xi_{1}, \xi_{2}\right)=\min \left(F_{c}\left(\xi_{1}\right), F_{h}\left(\xi_{2}\right)\right) \text {. }
$$

In particular, if $c_{0}$ and $h_{0}$ are anticomonotonic (i.e. $c_{0}$ and $-h_{0}$ are comonotonic) and positive random variables on $(\Omega, \mathcal{F}, \mathbb{P})$ and if we denote by $F_{c_{0}}^{-1}$ and $F_{h_{0}}^{-1}$ the respective inverse distributions of $c_{0}$ and $h_{0}$. We have:

$$
\mathbb{E}\left(h_{0} c_{0}\right)=\int_{0}^{\infty} F_{c_{0}}^{-1}(t) F_{h_{0}}^{-1}(1-t) d t
$$

and for every $c$, distributed as $c_{0}$ :

$$
\mathbb{E}\left(h_{0} c_{0}\right) \leq \mathbb{E}\left(h_{0} c\right)
$$

Other characterizations of comonotonic random variables can be found in Denneberg [4]. In particular, if two random variables $c$ and $h$ are such that there exists a nondecreasing function $\varphi$ for which $c$ can be written in the form $c=\varphi(h)$ (or if $h$ can be written in the form $h=\varphi(c)$ ), then $c$ and $h$ are comonotonic. In fact, $c$ and $h$ are comonotonic if and only if they are nondecreasing functions of the same third random variable $x$, which can be chosen to be equal to $c+h$ (Denneberg [4], Proposition 4.5, p.54). Furthermore, when $c$ and $h$ are nondecreasing functions of $c+h$, it is clear that these functions can be chosen to be continuous and even 1-Lipschitz. Hence $c$ and $h$ are comonotonic if and only if they are continuous nondecreasing functions of the same third random variable $c+h$. Finally, from a computational point of view, a usefull property for positive comonotonic random variables $c$ and $h$ is that $\operatorname{cov}^{Q}(c, h) \geq 0$ for all probability measure $Q$ equivalent to $P$.

\subsection{Characterization of efficient trading strategies}

We are now able to state the following theorem which characterizes efficient contingent claims.

Theorem 3 Let $\left(x_{0}, q_{0}\right) \in \mathcal{K}$. A terminal wealth $c_{0} \in \mathcal{C}\left(x_{0}, q_{0}\right)$ is efficient for the initial wealth $x_{0}$, and the quantity $q_{0}$ of the contingent claim $f$ to hedge, if and only if there exists $\left(y_{0}, r_{0}\right) \in \mathcal{G}, h_{0} \in \mathcal{D}\left(y_{0}, r_{0}\right)$ such that $\mathbb{P}\left(h_{0}=0\right)=0$ and:

1. $h_{0}$ prices $c_{0}$, i.e., $\mathbb{E}\left(h_{0} c_{0}\right)=x_{0} y_{0}+q_{0} r_{0}$, with $x_{0}$ such that $c_{0} \in$ $\mathcal{C}\left(x_{0}, q_{0}\right)$. 
2. Random variables $c_{0}$ and $h_{0}$ are anticomonotonic.

3. Random variables $c_{0}$ and $h_{0}$ satisfy the following condition:

$$
\left\{\begin{array}{lll}
\text { ess } \sup c_{0}<+\infty & \Rightarrow & \text { ess inf } h_{0}>0 \\
\text { ess inf } c_{0}>0 & \Rightarrow & \text { ess } \sup h_{0}<+\infty
\end{array} .\right.
$$

Thus, a contingent claim $c_{0}$ is efficient if and only if there exists a positive linear pricing rule $h_{0}$ such that $c_{0}$ brings at least as much value as in cheaper states of the world. Another message of this theorem is the existence of a density $h_{0}$ effectively pricing the net efficient consumption bundle $c_{0}$, which is not the case for every contingent claims.

We will see in the following proof that item (1) and (2) of Theorem (3) derive directly from the existence of a saddle point for the optimization problem. Item (3) is more technical since it is a consequence of the fact that utility functions $U \in \mathcal{U}$ are defined on all $\mathbb{R}_{+}^{*}$ (and not on an interval I in $\mathbb{R}_{+}^{*}$ ).

Note that the introduction of the set $\mathcal{D}(y, r)$ for $(y, r)$ helps us to take into account the random endowment to hedge. With no random endowment, we obtain:

Corollary 3.1 Without random endowment, a contingent claim $c_{0} \in \mathcal{C}\left(x_{0}\right)$ for $x_{0}>0$ is efficient if and only if there exists $h_{0} \in \mathcal{D}(1)$ such that $\mathbb{P}\left(h_{0}=\right.$ $0)=0$ and:

1. $h_{0}$ prices $c_{0}$, i.e., $\mathbb{E}\left(h_{0} c_{0}\right)=x_{0}$ with $c_{0} \in \mathcal{C}\left(x_{0}\right)$.

2. Random variables $c_{0}$ and $h_{0}$ are anticomonotonic.

3. Random variables $c_{0}$ and $h_{0}$ satisfy the following condition:

$$
\left\{\begin{array}{lll}
\text { ess } \sup c_{0}<+\infty & \Rightarrow & \text { ess inf } h_{0}>0 \\
\text { ess inf } c_{0}>0 & \Rightarrow & \text { ess } \sup h_{0}<+\infty
\end{array} .\right.
$$

The general idea of the following demonstration is to obtain Theorem (3) from the convex optimization problem first order conditions as in Jouini and Kallal [14]. For this purpose we need the following duality result, the proof of which is provided in the Appendix.

Let an economic agent be defined by its utility function $U$ belonging to $\mathcal{U}$, an initial wealth $x_{0}$ and a terminal contingent claim $f$ to hedge in quantity $q_{0}$, with $\left(x_{0}, q_{0}\right) \in \mathcal{K}$. We consider the perturbed problem:

$$
u\left(x_{0}, q_{0}\right) \triangleq \sup _{c \in \mathcal{C}\left(x_{0}, q_{0}\right)} \mathbb{E}[U(c)] .
$$

One can easily see that $u$ is a concave and strictly increasing function with respect to the initial wealth. For $(y, r) \in \mathcal{G}$, we define the dual optimization problem as

$$
v(y, r) \triangleq \inf _{h \in \mathcal{D}(y, r)} \mathbb{E}(V(h))
$$


where $V: \mathbb{R}_{*}^{+} \mapsto \mathbb{R}$ is the Legendre-transform of the function $-U(-x)$ :

$$
V(y) \triangleq \sup _{x>0}[U(x)-x y] .
$$

$V$ is a decreasing, convex function satisfying $\lim _{y \rightarrow+\infty} V_{l}^{\prime}(y)=0$, where $V_{l}^{\prime}$ is the left derivative of $V$. By classical duality results, the following bidual relationship holds:

$$
U(x)=\inf _{y>0}[V(y)+x y] .
$$

We want to prove that there is no duality gap between the primal and dual problems. The following lemma states that the functions $u$ and $v$ are indeed conjugate.

Lemma 3.1 Assume that:

$$
u(x, q)<\infty \text { for some }(x, q) \in \mathcal{K} .
$$

We have:

1. $\mathcal{K} \subset \operatorname{dom}(u)$ and the value function $u$ and $v$ are conjugate:

$$
\begin{aligned}
& v(y, r)=\sup _{(x, q) \in \mathcal{K}}[u(x, q)-x y-q r] \text { for }(y, r) \in \mathcal{G}, \\
& u(x, q)=\inf _{(y, r) \in \mathcal{G}}[v(y, r)+x y+q r] \text { for }(x, q) \in \mathcal{K} .
\end{aligned}
$$

2. If $v(y, r)<+\infty$, then the optimal solution $\hat{h} \in \mathcal{D}(y, r)$ of the problem (2.4) exists.

We are now in a position to obtain the characterization of efficient contingent claims (Theorem (3)).

Proof of Theorem 3.

We begin by proving that efficient contingent claims satisfy the properties stated in the theorem. For a contingent claim satisfying these properties, we then study the converse assertion by constructing a utility function such that the contingent claim is solution of the problem of expected utility maximization.

Let us start with the direct implication. Let $c_{0} \in \mathcal{C}\left(x_{0}, q_{0}\right),\left(x_{0}, q_{0}\right) \in \mathcal{K}$ and suppose that $c_{0}$ is strictly efficient with the utility function $U$. Let $\left(y_{0}, r_{0}\right) \in$ $\partial u\left(x_{0}, q_{0}\right)$. Since $u$ and $v$ are conjuguate (see Lemma (3.1)) then:

$$
u\left(x_{0}, q_{0}\right)-v\left(y_{0}, r_{0}\right)=x_{0} y_{0}+q_{0} r_{0} .
$$

In particular, $v\left(y_{0}, r_{0}\right)<+\infty$. By Lemma (3.1), there exists $h_{0} \in \mathcal{D}\left(y_{0}, r_{0}\right)$ solution of the dual problem. Besides, we have:

$$
\begin{aligned}
u\left(x_{0}, q_{0}\right)=\mathbb{E}\left[U\left(c_{0}\right)\right] & \leq \mathbb{E}\left[V\left(h_{0}\right)+h_{0} c_{0}\right] \\
& \leq v\left(y_{0}, r_{0}\right)+x_{0} y_{0}+q_{0} r_{0} .
\end{aligned}
$$


Thus, the above inequalities become equalities and we obtain:

$$
\begin{aligned}
c_{0} & =\operatorname{argmax}_{\{c \geq 0\}} \mathbb{E}\left(U(c)-h_{0} c\right), \\
\mathbb{E}\left(h_{0} c_{0}\right) & =x_{0} y_{0}+q_{0} r_{0} .
\end{aligned}
$$

We claim now that $\mathbb{P}\left(h_{0}=0\right)$. Indeed, suppose the existence of a set $A$, with $\mathbb{P}(A) \neq 0$, such that $\left(h_{0}\right)_{\mid A}=0$. Let $\tilde{c}=c_{0}+1_{A}$. We have $\mathbb{E}(U(\tilde{c}))>$ $\mathbb{E}\left(U\left(c_{0}\right)\right)$, since $U$ is strictly increasing. Moreover, $\left.\mathbb{E}\left(h_{0} \tilde{c}\right)\right)=\mathbb{E}\left(h_{0} c_{0}\right)$, which is in contradiction with (2.10).

Furthermore, $c_{0}$ and $h_{0}$ are anticomonotonic. Indeed, with equation (2.10), there exists $A \in \mathcal{F}$ such that $\mathbb{P}(A)=1$ and, for every $\omega \in A$ :

$$
c_{0}(\omega)=\operatorname{argmax}_{x>0} U(c)-h_{0}(\omega) x .
$$

Therefore, if $\left(\omega_{1}, \omega_{2}\right) \in A^{2}$ then:

$$
\begin{aligned}
& U\left(c_{0}\left(\omega_{1}\right)\right)-U\left(c_{0}\left(\omega_{2}\right)\right) \geq h_{0}\left(\omega_{1}\right)\left[c_{0}\left(\omega_{1}\right)-c_{0}\left(\omega_{2}\right)\right], \\
& U\left(c_{0}\left(\omega_{2}\right)\right)-U\left(c_{0}\left(\omega_{1}\right)\right) \geq h_{0}\left(\omega_{1}\right)\left[c_{0}\left(\omega_{2}\right)-c_{0}\left(\omega_{1}\right)\right] .
\end{aligned}
$$

Therefore, if we add up the previous inequalities, we obtain:

$$
\left[c_{0}\left(\omega_{2}\right)-c_{0}\left(\omega_{1}\right)\right]\left[h_{0}\left(\omega_{2}\right)-h_{0}\left(\omega_{1}\right)\right] \leq 0
$$

for every $\left(\omega_{1}, \omega_{2}\right) \in A^{2}$.

In order to conclude the proof of the first implcation, let us prove that:

$$
\begin{aligned}
\text { ess } \sup c_{0}<+\infty & \Rightarrow \text { ess } \inf h_{0}>0 \\
\text { ess inf } c_{0}>0 & \Rightarrow \text { ess } \sup h_{0}<+\infty
\end{aligned}
$$

Assume for a moment that ess $\sup c_{0}=x_{0} \in \mathbb{R}_{+}<+\infty$ and ess inf $h_{0}=0$. Let $x_{s}>x_{0}$ and $\varepsilon>0$ be such that $\varepsilon \leq \frac{U\left(x_{s}\right)-U\left(x_{0}\right)}{x_{s}-x_{0}}$, which is always possible because $U$ is strictly increasing. By concavity of $U$, for every $x \leq x_{0}$, we have:

$$
\varepsilon \leq \frac{U\left(x_{s}\right)-U(x)}{x_{s}-x}
$$

Define then $A \triangleq\left\{h_{0} \leq \frac{\varepsilon}{2}\right\}$ and $\tilde{c} \triangleq c_{0} 1_{A}^{c}+x_{s} 1_{A}$. First, note that $\mathbb{P}(A) \neq 0$ because ess $\inf h_{0}=0$. On the one hand, we obtain with the relationship (2.10):

$$
\mathbb{E}(U(\tilde{c}))-\mathbb{E}\left(U\left(c_{0}\right)\right) \leq \mathbb{E}\left(h_{0}\left(\tilde{c}-c_{0}\right)\right) \leq \frac{\varepsilon}{2} \mathbb{E}\left(\left(x_{s}-c_{0}\right) \mathbf{1}_{A}\right) .
$$

On the other hand:

$$
\begin{aligned}
\mathbb{E}(U(\tilde{c}))-\mathbb{E}\left(U\left(c_{0}\right)\right) & =\mathbb{E}(\underbrace{\left(U\left(x_{s}\right)-U\left(c_{0}\right)\right) \mathbf{1}_{A}}_{\substack{a_{s . s} \\
\geq}\left(x_{s}-c_{0}\right) \mathbf{1}_{A}}) \\
& \geq \varepsilon \mathbb{E}\left(\left(x_{s}-c_{0}\right) \mathbf{1}_{A}\right)
\end{aligned}
$$

which is in contradiction with $(2.12)$ because $\mathbb{E}\left(\left(x_{s}-c_{0}\right) \mathbf{1}_{A}\right) \neq 0$.

Now, suppose that ess inf $c_{0}=x_{0}>0$ and ess sup $h_{0}=+\infty$. Let us work towards a contradiction. Let $x_{1}$ be such that $0<x_{1}<x_{0}$. We can choose a 
sequence $\left(\omega_{n}\right)_{\{n \geq 1\}}$ such that $h_{0}\left(\omega_{n}\right)$ is increasing and $\lim _{n \rightarrow+\infty} h_{0}\left(\omega_{n}\right)=+\infty$. Since $h_{0}\left(\omega_{n}\right) \in \partial U\left(\left(c_{0}\left(\omega_{n}\right)\right)\right.$, we have that $c_{0}\left(\omega_{n}\right)$ is a decreasing sequence and:

$$
U\left(x_{1}\right)-U\left(c_{0}\left(\omega_{n}\right)\right) \leq h_{0}\left(\omega_{n}\right)\left(x_{1}-c_{0}\left(\omega_{n}\right)\right) \leq h_{0}\left(\omega_{n}\right)\left(x_{1}-x_{0}\right),
$$

and thus by monotonicity of $U($.$) , we have:$

$$
U\left(x_{1}\right)-U\left(c_{0}\left(\omega_{1}\right)\right) \leq U\left(x_{1}\right)-U\left(c_{0}\left(\omega_{n}\right)\right) \leq h_{0}\left(\omega_{n}\right)\left(x_{1}-x_{0}\right)
$$

where the last term of this inequality goes to $-\infty$. We conclude that $U\left(x_{1}\right)=-\infty$, which is a contradiction with $\mathbb{R}_{+}^{*} \subset \operatorname{dom}(U)$.

We turn now to the proof of the converse implication. Assume there exists $\left(y_{0}, r_{0}\right) \in \mathbb{R}_{+}^{2}, h_{0} \in \mathcal{D}\left(y_{0}, r_{0}\right)$ and $c_{0} \in \mathcal{C}\left(x_{0}, q_{0}\right)$ which satisfy the three properties of the theorem. Let's build a utility function $U \in \mathcal{U}$ such that $c_{0}$ is efficient for $U$. Let $A$, with probability one, be such that for every $\left(\omega_{1}, \omega_{2}\right) \in A^{2}$, we have:

$$
\left[c_{0}\left(\omega_{1}\right)-c_{0}\left(\omega_{2}\right)\right]\left[h_{0}\left(\omega_{1}\right)-h_{0}\left(\omega_{2}\right)\right] \leq 0
$$

and such that, for every $w \in A, h_{0}(\omega) \leq \operatorname{ess} \sup h_{0}$. We define $h: \mathbb{R}^{+} \rightarrow \overline{\mathbb{R}}_{+}$by:

$$
h(x)=\left\{\begin{array}{ll}
\inf _{A}\left\{h_{0}(\omega) \mid c_{0}(\omega) \leq x\right\} & \text { if }\left\{c_{0}(\omega) \leq x\right\} \neq \emptyset \\
\operatorname{ess} \sup h_{0} & \text { otherwise }
\end{array} .\right.
$$

The function $h$ satisfies the following properties.

First, $h$ is non increasing and $h\left(\mathbb{R}_{+}^{*}\right) \subset \mathbb{R}_{+}^{*}$. Indeed, $h$ is obviously non increasing. Moreover if $x<\operatorname{ess} \sup c_{0}$ then $h(x)$ is not equal to zero. Indeed, if $h(x)=0$ then $\left\{c_{0}>x\right\} \subset\left\{h_{0}=0\right\}$. It follows that $\mathbb{P}\left(h_{0}=0\right) \neq 0$, which is in contradiction with the hypothesis. In the same way, we prove that if $x>\operatorname{ess} \inf c_{0}$ then $h(x)<+\infty$. Thus, the last equation implies that $h\left(\mathbb{R}_{+}^{*}\right) \subset \mathbb{R}_{+}^{*}$.

Second, we have $h\left(c_{0}\right) \leq h_{0}$ and $\left(x<c_{0} \Rightarrow h(x) \geq h_{0}\right)$. The first statement follows directly from the definition of $h$. Suppose there exists $x<c_{0}(\omega)$ such that $h(x)<h_{0}(\omega)$. Then there exists $\omega^{\prime} \in A$ such that $c_{0}\left(\omega^{\prime}\right) \leq x<c_{0}(\omega)$ and $h_{0}\left(\omega^{\prime}\right)<h_{0}(\omega)$ which is in contradiction with equation (2.13).

Let's define the utility function $U(x) \triangleq \int_{1}^{x} h(t) d t$ for every $x \in \mathbb{R}_{+}^{*}$. From the properties of $h$, we deduce that $U$ is strictly increasing and concave. Let $c \in$ $\mathcal{C}\left(x_{0}, q_{0}\right)$ be strictly positive. We obtain:

$$
\mathbb{E}(U(c))-\mathbb{E}\left(U\left(c_{0}\right)\right)=\mathbb{E}(\underbrace{\int_{c^{*}}^{c} h(t) d t}_{\substack{a . s . \\ \leq h_{0}\left(c-c_{0}\right)}}) \leq \mathbb{E}\left(h_{0}\left(c-c_{0}\right)\right) .
$$

Moreover, $\mathbb{E}\left(h_{0} c\right) \leq x_{0} y_{0}+q_{0} r_{0}$ and $\mathbb{E}\left(h_{0} c_{0}\right)=x_{0} y_{0}+q_{0} r_{0}$, we have:

$$
\mathbb{E}(U(c))-\mathbb{E}\left(U\left(c_{0}\right)\right) \leq 0
$$

which proves that $c_{0}$ is efficient. 


\subsection{Inefficiency size}

Now that we can characterize efficient and inefficient trading strategies, we would like to evaluate the size of their inefficiency, that is, to have a measure of how far they are from being optimal. We follow the definition of Jouini and Kallal [14]:

Definition 4 Let $\left(x_{0}, q_{0}\right) \in \mathbb{R}_{+}^{2}$ and $c_{0} \in \mathcal{X}\left(x_{0}, q_{0}\right)$. For a given random endowment $f$ to hedge at date $T$, in quantity $q_{0}$, the inefficiency cost of the terminal wealth $c_{0} \in \operatorname{Adm}\left(q_{0}, f\right)$ is the difference $x_{0}-V_{q_{0} f}\left(c_{0}\right)$, where $V_{q_{0} f}\left(c_{0}\right)=\sup _{U \in \mathcal{U}} \inf _{c \in A d m\left(q_{0}, f\right)}\left\{\pi_{q_{0} f}(c) \mid \mathbb{E}\left(U(c)-U\left(c_{0}\right)\right) \geq 0\right\}$ is the "utility price" of $c_{0}$.

Indeed, $V_{q_{0}}\left(c_{0}\right)$ is the largest amount of money that is required by rational agents, with an uncertain future endowment $f$ to hedge, in order to get the same utility level as with the consumption bundle $c_{0}$. Hence, $\pi_{q_{0} f}\left(c_{0}\right)-V_{q_{0} f}\left(c_{0}\right)$ is the smallest discrepancy, across all rational agents with future endowment $f$ to hedge, between the cost of obtaining $c_{0}$ and the price at which it would be an optimal choice. Moreover, note that $\pi_{q_{0} f}\left(c_{0}\right) \geq V_{q_{0} f}\left(c_{0}\right)$, which means that the measure of inefficiency is always nonnegative.

In the case of a dynamically complete market, with a unique linear pricing rule $h_{0}$, the "utility price" coincides with the "distributional price" introduced by Dybvig [6]. Dybvig defined the distributional price of a contingent claim $c_{0}$ as the minimum cost to achieve the same distribution of consumption. However, in the case of markets with frictions (with a finite number of states of the world), where the pricing rule is defined as the supremum over a set of linear functions, Jouini and Kallal [14] show that the distributional price does not coincide with the utility price anymore. They prove in particular that the utility price of a contingent claim is the minimum cost over the convex hull of random variables with the same distribution as $c_{0}$.

We will now study how the results of Jouini and Kallal [14] may be extended to our context. We are going to prove in this section the following result:

Theorem 4 For a given contingent claim $f$ in quantity $q_{0}$ to hedge, the utility price of a consumption bundle $c_{0}$ satisfies:

1.

$$
\begin{aligned}
V_{q_{0} f}\left(c_{0}\right) & =\min _{c \in \operatorname{Adm}\left(q_{0}, f\right)}\left\{\pi_{q_{0} f}(c) \mid \mathbb{E}(U(c)) \geq \mathbb{E}\left(U\left(c_{0}\right)\right) \text { for all } U \in \mathcal{U}\right\} \\
& =\min _{c \in \operatorname{Adm}\left(q_{0}, f\right)}\left\{\pi_{q_{0} f}(c) \mid c \text { is a convex comb. of bundles distributed as } c_{0}\right\}
\end{aligned}
$$

2. $V_{q_{0} f}\left(c_{0}\right)=\sup _{p \in \tilde{\mathcal{P}}} \sup \left(P_{q_{0} . f}\left(c_{0}, h\right)-q_{0} p \mid h \in \mathcal{D}(1, p)\right)$ where

$$
P_{q_{0} f}\left(c_{0}, h\right)=\min _{c \in \operatorname{Adm}(q, f)}\left\{\mathbb{E}(h c) \mid c \text { distributed as } c_{0}\right)
$$


is the utility price of $c_{0}$ in the frictionless economy defined by the linear pricing rule $h$.

3. $V_{q_{0} f}\left(c_{0}\right)=\sup _{p \in \mathcal{P}} \sup _{h \in \mathcal{D}(1, p)}\left\{\int_{0}^{1} F_{c_{0}}^{-1}(y) F_{h}^{-1}(1-y) d y-q_{0} p\right\}$ where $F_{c_{0}}$ (respectively $F_{h}$ ) is the cumulative distribution function ${ }^{3}$ of $c_{0}$ (respectively of $h$ ), and $F^{-1}($.$) is the quantile function, defined as$ $F^{-1}(y)=\min \{z \mid F(z) \geq y\}$ for every $y \in(0,1)$. Moreover, if ess inf $c_{0}=$ 0 , the supremum over $\mathcal{D}(1, p)$ for $p \in \mathcal{P}$ becomes a maximum, i.e. there exists $\tilde{p}_{0} \in \tilde{\mathcal{P}}$ and $\tilde{h}_{0} \in \mathcal{D}\left(1, \tilde{p}_{0}\right)$, such that:

$$
V_{q_{0} f}\left(c_{0}\right)=\int_{0}^{1} F_{c_{0}}^{-1}(t) F_{\tilde{h}_{0}}^{-1}(1-t) d t-q_{0} \tilde{p}_{0}
$$

Result (1) says that the utility price of the net consumption bundle $c_{0}$ obtained after hedging the random endowment $q_{0} f$ is equal to the cost of the cheapest trading strategy leading to a net consumption bundle that is a convex combination of random variables with the same distribution as $c_{0}$. In particular, the utility price $V_{q_{0} f}\left(c_{0}\right)$ only depends on the distribution of $c_{0}$. Note that in the frictionless case, because the pricing $\pi$ is linear, the value

$\min _{c \in \operatorname{Adm}\left(q_{0}, f\right)}\left\{\pi_{q_{0} f}(c) \mid c\right.$ is a convex combination of bundles distributed as $\left.c_{0}\right\}$

is attained for a consumption bundle that has the same distribution as $c_{0}$. Hence, the utility price coincides with the minimum cost of achieving a given distribution of net consumption. In imperfect markets, though, this minimum is only attained for convex combination of consumption bundles that have the same distribution as $c_{0}$.

Result (2) states that the utility price $V_{q 0 f}\left(c_{0}\right)$ is the largest of the utility prices of $c_{0}$ in the underlying frictionless economies.

Note that in our general context, we must first solve problems of the existence of solutions. In particular we will show that the property ess inf $c_{0}=$ 0 , implying that the supremum over the set of linear pricing rules is a maximum, is strongly related to the proof of the existence of a dual solution $\hat{h}(y, r)$ for the dual problem $v(y, r)$ of utility maximization. Indeed, the proof of the existence of $\hat{h}(y, r)$ uses in a crucial way the assumption that utility functions are well defined on $\mathbb{R}_{+}^{*}$.

We are now going to prove this result. For this purpose we will need the following definitions and Lemma. For $c_{0} \in L_{+}^{1}$, a natural set we need to

\footnotetext{
${ }^{3}$ The cumulative distribution function $F_{c}(z)$ of a random variable $c$ defined on a probability space $(\Omega, \mathcal{F}, \mathbb{P})$ is the probability that the random variable $c$ is less than or equal to $z$ :

$$
F_{c}(z)=\mathbb{P}(c \leq x)
$$
}


study is the set of terminal wealth preferred by every agent whatever his or her preferences are, and defined by:

$$
\mathcal{P}\left(c_{0}\right) \triangleq\left\{c \in L_{+}^{1} \mid \mathbb{E}\left(U(c)-U\left(c_{0}\right)\right) \geq 0 \text { for every } U \in \mathcal{U}\right\} .
$$

We first need to characterize the set $\mathcal{P}\left(c_{0}\right)$, which is the set of contingent claims $c$ in $L_{+}^{1}$ such that $c \succeq_{2} c_{0}$ with respect to the so-called second order stochastic dominance. To do so, we denote by $\Sigma_{0}\left(c_{0}\right)$ the convex hull (closed with respect to the topology of convergence in probability) of the set of random variables distributed as $c_{0}$.

The following properties, characterize the second order stochastic dominance. Second order stochastic dominance has been in particular studied by Rothschild and Stiglitz in a couple of articles [17, 18]. Jouini and Kallal [14] suggest another charaterization of second order stochastic dominance, based upon $\Sigma_{0}\left(c_{0}\right)$.

Proposition 1 (Characterization of $\mathcal{P}\left(c_{0}\right)$ ). Let $c_{0}$ and $c$ be two random variables belonging to $L_{+}^{1}$. The following statements are equivalent:

1. $c \in \mathcal{P}\left(c_{0}\right)$,

2. $c \in \Sigma_{0}\left(c_{0}\right)+L_{+}^{1}$,

3. $\int_{0}^{x} \mathbb{P}(c \geq s) d s \geq \int_{0}^{x} \mathbb{P}\left(c_{0} \geq s\right) d s$ for every $x \in \mathbb{R}^{+}$.

Finally, Diamond and Stiglitz [5] introduce the single-crossing property, which is an useful criterion to prove second order stochastic dominance.

Proposition 2 Furthermore, if $c_{1}$ in $L_{+}^{1}$ is such that $\mathbb{E}\left(c_{1}\right)=\mathbb{E}\left(c_{0}\right)$ and such that there exists $x_{0} \geq 0$ satisfying (single-crossing property):

$$
\left\{\begin{array}{l}
\mathbb{P}\left(c_{1} \leq x\right) \leq \mathbb{P}\left(c_{0} \leq x\right) \text { for every } x \leq x_{0} \\
\mathbb{P}\left(c_{1} \leq x\right) \geq \mathbb{P}\left(c_{0} \leq x\right) \text { for every } x>x_{0}
\end{array}\right.
$$

Then $c_{1}$ belongs to $\mathcal{P}\left(c_{0}\right)$.

With the next Lemma (4.1) we will be able to compute the utility price of a contingent claim as the supremum over the set of utility prices in each frictionless economy defined with a linear pricing rule $h \in \mathcal{D}(1, p)$, for every $p \in \tilde{\mathcal{P}}$. Note that the following lemma states that there exists a contingent claim preferred by every agent with a funding cost of $\inf _{c \in \mathcal{P}\left(c_{0}\right)} \pi_{q_{0} f}(c)$. The proof of this Lemma is provided in the Appendix.

Lemma 4.1 Let $c_{0} \in \operatorname{Adm}\left(q_{0}, f\right)$. We have :

$$
\begin{aligned}
\inf _{c \in \mathcal{P}\left(c_{0}\right)} \pi_{q_{0} f}(c) & =\min _{c \in P\left(c_{0}\right)} \sup _{p \in \mathcal{P}} \sup _{h \in \mathcal{D}(1, p)} \mathbb{E}(h c)-q_{0} p \\
& =\sup _{p \in \mathcal{P}} \sup _{h \in \mathcal{D}(1, p)} \min _{c \in \mathcal{P}\left(c_{0}\right)} \mathbb{E}(h c)-q_{0} p .
\end{aligned}
$$


We are now in a position to compute the inefficiency size of a terminal wealth.

Proof of Theorem 4.

We begin to study the case with a linear pricing rule; then using Lemma (4.1), we extend the result to the general case.

Let $h \in \mathcal{D}(1, p)$ with $\mathbb{P}(h=0)=0$. We first prove that:

$$
\sup _{U \in \mathcal{U}} \inf _{c \in B^{U}\left(c_{0}\right)} \mathbb{E}(h c)=\min _{c \in \mathcal{P}\left(c_{0}\right)} \mathbb{E}(h c)
$$

with $B^{U}\left(c_{0}\right) \triangleq\left\{c \geq 0 \mid \mathbb{E}(U(c)) \geq \mathbb{E}\left(U\left(c_{0}\right)\right)\right\}$. Indeed, since the probability space is atomless, we can choose $\tilde{c}$ with the same distribution as $c_{0}$ such that $\tilde{c}$ is anticomonotonic with $h$. Since $\tilde{c} \in \mathcal{P}\left(c_{0}\right)$, we deduce the following inequality:

$$
\sup _{U \in \mathcal{U}} \inf _{c \in \mathcal{B}_{U}\left(c_{0}\right)} \mathbb{E}(h c) \leq \mathbb{E}(h \tilde{c}) .
$$

It could happen that $\tilde{c}$ and $h$ don't satisfy the last condition of Theorem (3), that is:

$$
\begin{aligned}
\text { ess } \sup \tilde{c}<+\infty & \Rightarrow \operatorname{ess} \inf h \neq 0, \\
\text { ess } \inf \tilde{c}>0 & \Rightarrow \quad \operatorname{ess} \sup h \neq+\infty .
\end{aligned}
$$

Nevertheless, we consider for $\varepsilon>0$, and $x>0$ the random variables:

$$
c_{x, \varepsilon}=\exp (-\varepsilon h) \tilde{c} \mathbf{1}_{\{\tilde{c} \leq x\}}+\left[\tilde{c}+\frac{\varepsilon}{1+h}\right] \mathbf{1}_{\{\tilde{c}>x\}} .
$$

The random variables $c_{x, \varepsilon}$ and $h$ are anticomonotonic and satisfy the last condition of Theorem (3). Since $\lim _{x \rightarrow+\infty} \mathbb{E}\left(c_{x, \varepsilon}\right)=\mathbb{E}(\exp (-\varepsilon h) \tilde{c})<\mathbb{E}(\tilde{c})$, and $\lim _{x \rightarrow 0} \mathbb{E}\left(c_{x, \varepsilon}\right)=\mathbb{E}\left(\tilde{c}+\frac{\varepsilon}{1+h}\right)>\mathbb{E}(\tilde{c})$, one can choose $x(\varepsilon)$, such that $\mathbb{E}\left(c_{\varepsilon, x(\varepsilon)}\right)=$ $\mathbb{E}(\tilde{c})$. The random variables $c_{\varepsilon, x(\varepsilon)}$ and $h$ satisfy the last condition of Theorem (3): $c_{\varepsilon, x(\varepsilon)}$ is efficient and there exists a utility function $U_{\varepsilon} \in \mathcal{U}$ such that:

$$
\mathbb{E}\left(h c_{\varepsilon, x(\varepsilon)}\right)=\min _{c \in B^{U_{\varepsilon}}} \mathbb{E}(h c) .
$$

Therefore:

$$
\begin{aligned}
\inf _{c \in \mathcal{P}\left(c_{\varepsilon, x(\varepsilon)}\right)} E(h c) & =\sup _{U \in \mathcal{U}} \inf _{c \in \mathcal{B}^{U}\left(c_{\varepsilon, x(\varepsilon)}\right)} \mathbb{E}(h c) \\
& =\inf _{c \in \mathcal{B}^{U} \varepsilon} \mathbb{E}(h c) \\
& =\mathbb{E}\left(h c_{\varepsilon, x(\varepsilon)}\right) .
\end{aligned}
$$

The random variables $c_{\varepsilon, x(\varepsilon)}$ have been constructed in order to verify :

$$
\begin{aligned}
& \mathbb{P}\left(c_{\varepsilon, x(\varepsilon)} \leq x\right) \geq \mathbb{P}(\tilde{c} \leq x) \text { for all } x \leq x(\varepsilon), \\
& \mathbb{P}\left(c_{\varepsilon, x(\varepsilon)} \leq x\right) \geq \mathbb{P}(\tilde{c} \leq x) \text { elsewhere. }
\end{aligned}
$$

Thus, the single-crossing property implies that $\tilde{c} \in \mathcal{P}\left(c_{\varepsilon, x(\varepsilon)}\right)$, and in consequence:

$$
\begin{aligned}
\sup _{U \in \mathcal{U}} \inf _{c \in B^{U}(\tilde{c})} \mathbb{E}(h c) & \geq \sup _{U \in \mathcal{U}} \inf _{c \in B^{U}\left(c_{\varepsilon, x(\varepsilon)}\right)} \mathbb{E}(h c) \\
& =\mathbb{E}\left(h c_{\varepsilon, x(\varepsilon)}\right) .
\end{aligned}
$$


Then, with the help of the dominated convergence Theorem, $\lim _{\varepsilon \rightarrow 0} \mathbb{E}\left(h c_{\varepsilon, x(\varepsilon)}\right)=$ $\mathbb{E}(h \tilde{c})$, and we obtain:

$$
\sup _{U \in \mathcal{U}} \inf _{c \in B^{U}(\tilde{c})} \mathbb{E}(h c) \geq \lim _{\varepsilon \rightarrow 0} \mathbb{E}\left(h c_{\varepsilon, x(\varepsilon)}\right)=\mathbb{E}(h \tilde{c}) .
$$

We deduce that:

$$
\sup _{U \in \mathcal{U}} \inf _{c \in B^{U}(\tilde{c})} \mathbb{E}(h c) \geq \min _{c \in \mathcal{P}\left(c_{0}\right)} \mathbb{E}(h c)=\mathbb{E}(h \tilde{c})
$$

and therefore:

$$
\sup _{U \in \mathcal{U}} \inf _{c \in B^{U}(\tilde{c})} \mathbb{E}(h c)=\min _{c \in \mathcal{P}\left(c_{0}\right)} \mathbb{E}(h c) .
$$

Let us now consider the general case. We begin with straightforward assertions. We recall that:

$$
\left.V_{q_{0} f}\left(c_{0}\right)=\sup _{U \in \mathcal{U}} \inf _{c \in B_{U}\left(c_{0}\right)} \pi(c)=\sup _{U \in \mathcal{U}} \sup _{p \in \tilde{\mathcal{P}}} \sup _{h \in \mathcal{D}(1, p)} \inf _{c \in B_{U}\left(c_{0}\right)} \mathbb{E}(h c)\right)-q_{0} p
$$

and in consequence:

$$
V_{q_{0} f}\left(c_{0}\right) \geq \sup _{p \in \tilde{\mathcal{P}}}\left\{\begin{array}{l}
\left.\sup _{h \in \mathcal{D}(1, p)} \sup _{U \in \mathcal{U}} \inf _{c \in B_{U}\left(c_{0}\right)} \mathbb{E}(h c)\right)-q_{0} p . \\
\mathbb{P}(h=0)=0
\end{array}\right.
$$

But applying the result of the first step, we have:

$\sup _{p \in \tilde{\mathcal{P}}}\left\{\begin{array}{l}\left.\sup _{h \in \mathcal{D}(1, p)} \sup _{U \in \mathcal{U}} \inf _{c \in B_{U}\left(c_{0}\right)} \mathbb{E}(h c)\right)-q_{0} p=\sup _{p \in \tilde{\mathcal{P}}}\left\{\begin{array}{l}\sup _{h \in \mathcal{D}(1, p)} \\ \mathbb{P}(h=0)=0\end{array} \min _{c \in \mathcal{P}\left(c_{0}\right)} \mathbb{E}(h c)-q_{0} p .\right. \\ \mathbb{P}(h=0)=0\end{array}\right.$

Furthermore, let $h^{*} \in \mathcal{D}\left(1, p^{*}\right)$, with $\mathbb{P}\left(h^{*}=0\right) \neq 0$ for some $p^{*} \in \mathcal{P}$. If we consider the sequence $h_{n}=\frac{1}{n} h^{*}+\frac{n-1}{n} h$ for some $h \in \mathcal{D}(1, p), h_{n}$ belongs to $\mathcal{D}\left(1, \frac{1}{n} p^{*}+(1-\right.$ $\left.\left.\frac{1}{n}\right) p\right)$, and $\mathbb{P}\left(h_{n}=0\right) \neq 0$. We have for every $c \in \mathcal{P}\left(c_{0}\right), \lim _{n \rightarrow+\infty} \mathbb{E}\left(h_{n} c\right)=\mathbb{E}\left(h^{*} c\right)$.

Thus,

$$
\inf _{c \in \mathcal{P}\left(c_{0}\right)} \mathbb{E}\left(h^{*} c\right)-q_{0} p^{*} \leq \sup _{p \in \tilde{\mathcal{P}}}\left\{\begin{array}{l}
\left.\sup _{h \in \mathcal{D}(1, p)} \min _{c \in \mathcal{P}\left(c_{0}\right)} \mathbb{E}(h c)\right)-q_{0} p \\
\mathbb{P}(h=0)=0
\end{array}\right.
$$

We deduce that:

$$
\sup _{p \in \tilde{\mathcal{P}}}\left\{\begin{array}{l}
\left.\left.\sup _{h \in \mathcal{D}(1, p)} \min _{c \in \mathcal{P}\left(c_{0}\right)} \mathbb{E}(h c)\right)-q_{0} p=\sup _{p \in \mathcal{P}} \sup _{h \in \mathcal{D}(1, p)} \min _{c \in \mathcal{P}\left(c_{0}\right)} \mathbb{E}(h c)\right)-q_{0} p . \\
\mathbb{P}(h=0)=0
\end{array}\right.
$$

Now, we can use Lemma (4.1), and obtain the following inequality:

$$
V_{q_{0} f}\left(c_{0}\right) \geq \min _{c \in \mathcal{P}\left(c_{0}\right)} \sup _{p \in \tilde{\mathcal{P}}} \sup _{h \in \mathcal{D}(1, p)} \mathbb{E}(h c)-q_{0} p .
$$

The other inequality is straightforward and we conclude that:

$$
\begin{aligned}
V_{q_{0} f}\left(c_{0}\right) & =\min _{c \in \mathcal{P}\left(c_{0}\right)} \pi_{q_{0} f}(c) \\
& =\sup _{p \in \tilde{\mathcal{P}}} \sup _{h \in \mathcal{D}(1, p)} \min _{c \in \mathcal{P}\left(c_{0}\right)} \mathbb{E}(h c)-q_{0} p .
\end{aligned}
$$


It remains to prove that the infinimum is attained as soon as ess inf $c_{0}=0$.

Let us finally prove that suprema are maxima as soon as ess inf $c_{0}=0$. Indeed, choose $p_{n} \in \mathcal{P}$, and $h_{n} \in \mathcal{D}\left(1, p_{n}\right)$ such that:

$$
V_{q_{0} f}\left(c_{0}\right)=\lim _{n \rightarrow+\infty} \int_{0}^{1} F^{-} 1_{h_{n}}(t) F_{c_{0}}^{-1}(1-t) d t-q_{o} p_{n} .
$$

With the help of Komlos Theorem, one can find a maximizing sequence $\left(p_{n}, h_{n}\right)$, with $p_{n} \rightarrow p_{0}$ and $h_{n} \in \mathcal{D}\left(1, p_{n}\right)$ such that $h_{n} \rightarrow h_{0}$ with $h_{0} \in \mathcal{D}\left(1, p_{0}\right)$.

Let's prove:

$$
\lim _{n \rightarrow+\infty} \int_{0}^{1} F_{h_{n}}^{-1}(t) F_{c_{0}}^{-1}(1-t) d t-q_{0} p_{n}=\int_{0}^{1} F_{h_{0}}^{-1}(t) F_{c_{0}}^{-1}(1-t) d t-q_{0} p_{0} .
$$

Indeed, let $\varepsilon>0$ and, since inf ess $c_{0}=0$, let $t_{0}$ be such that $F_{c_{0}}^{-1}(t)<\varepsilon$ if $t \leq t_{0}$. Then $\int_{0}^{t_{0}} F_{c_{0}}^{-1}(t) F_{h_{n}}^{-1}(1-t) d t<\varepsilon$. Moreover, for every $t \geq t_{0}$ :

$$
F_{h_{n}}^{-1}(1-t) \leq F_{h_{n}}^{-1}\left(1-a_{0}\right) \leq \frac{\int_{0}^{1} F_{h_{n}}^{-1}(1-t) d t}{a_{0}}=\frac{1}{a_{0}} .
$$

Then, with the dominated convergence Theorem, we obtain the existence of $n$ such that for every $n \geq n_{0},\left|\int_{a_{0}}^{1}\left[F_{h_{n}}^{-1}(1-t)-F_{h_{0}}^{-1}(1-t)\right] F_{c_{0}}^{-1}(t) d t\right| \leq \varepsilon$ and the result follows. Therefore, the theorem is proved.

\section{Applications}

In this section we provide several applications of the results of the previous sections. First, we prove that the superreplication strategies that are known to be too expensive in the presence of market frictions have a zeroinefficiency size. Roughly speaking, this means that it is always possible to find individuals that are willing to adopt strategies arbitrarily close to the superreplication one. As in Dybvig [6] or Jouini and Kallal [14], we also provide applications of our results for performance measurement.

\subsection{Trading strategies with no inefficiency size}

In this paragraph, we study the strategies with no inefficiency size. An efficient trading strategy has obviously no inefficiency size, but the converse is not true in general. We would like now to have a simple characterization of strategies with no efficiency cost. We begin with the following lemma, which states that one can study only the class of random variables $c$ such that ess inf $c=0$ :

Lemma 5.1 Let $c_{0}$ be a consumption bundle with no inefficiency size, and $x_{0}$ its price. Let $b_{0}$ be a cash endowment, i.e. a constant random variable. Then the contingent claim $c_{0}+b_{0}$ has no inefficiency size for the initial portfolio $x_{0}+b_{0}$. 
Proof of Lemma 5.1.

Indeed, suppose that $c_{0}+b_{0}$ has an inefficiency size. From Theorem (4), there exists a strategy which leads to the consumption bundle $\tilde{c}$ belonging to the closed convex set of random vectors distributed as $c_{0}+b_{0}$ from an initial portfolio $\tilde{x}$, with $\tilde{x}<x_{0}+b_{0}$. Let $\tilde{c}_{1}=\tilde{c}-b_{0}$. The random variable $\tilde{c}$ belongs to the set $\Sigma_{0}\left(c_{0}\right)$, and is attainable from the initial portfolio $\tilde{x}-b_{0}$. This implies in particular that $c_{0}$ is inefficient with respect to the initial portfolio $x_{0}$, which is a contradiction, and concludes the proof.

In a context of financial markets with a finite number of states one can see that efficient consumption bundles are stable with the addition of a constant (see Jouini and Kallal [14]). Note that, in our framework, this is not the case anymore, because of the last properties of Theorem (3).

One can furthermore obtain the following characterization for the contingent claims with no inefficiency size.

Theorem 5 For a random endowment $f$, in quantity $q_{0}$, a consumption bundle $c_{0} \in \operatorname{Adm}\left(q_{0}, f\right)$, s.t. ess inf $c=0$ has no inefficiency size if and only if there exists $p_{0} \in \mathcal{D}(1, p)$ and $h_{0} \in \mathcal{D}(1, p)$, which satisfies:

1. $\mathbb{E}\left(h_{0} c\right)-q_{0} p_{0}=\pi_{q_{0} f}(c)$

2. the random variables $h_{0}$ and $c_{0}$ are anticomonotonic.

Proof of Theorem 5.

Suppose that $c_{0} \in \operatorname{Adm}\left(q_{0}, f\right)$ verifies the properties of Theorem (5). We have:

$$
\pi_{q_{0} f}(c)=\mathbb{E}\left(h_{0} c_{0}\right)-q_{0} p_{0}=\int_{0}^{1} F_{h_{0}}^{-1}(t) F_{c_{0}}^{-1}(1-t) d t-q_{0} p_{0} .
$$

Moreover, for all $h \in \mathcal{D}(1, p)$ and $p \in \mathcal{P}$, we have the following result:

$$
\pi_{q_{0} f}\left(c_{0}\right)=\mathbb{E}\left(h_{0} c_{0}\right)-q_{0} p_{0} \geq \mathbb{E}\left(h c_{0}\right)-q_{0} p \geq \int_{0}^{1} F_{h}^{-1}(t) F_{c_{0}}^{-1}(1-t) d t-q_{0} p
$$

and therefore, with the last theorem, there is no inefficiency cost for $c_{0}$.

Note that, in this theorem, the random variable of pricing $h_{0}$ is not necessarily strictly positive as it was the case for the characterization of strictly efficient consumption bundles. Let us give now two applications of the above result, first in the case of no consumption bundle to hedge, and then for the superreplication strategy.

Corollary 5.1 A contingent claim with ess inf $c=0$ has no inefficiency cost if and only if there exists $h \in \mathcal{D}(1)$, which satisfies: 
1. $\mathbb{E}(h c)=\pi(c)$

2. $c$ and $h$ are anticomonotonic.

This corollary is an immediate consequence of the last theorem. Another corollary quite interesting, is that superreplication strategies have a zeroinefficiency size:

Corollary 5.2 Let $f$ be a contingent claim with $f \leq 0$. The superreplication strategy of the contingent claim $f$ has no inefficiency size.

Proof of Corollary 5.2.

Indeed, let $X \in \mathcal{X}(\hat{x})$ be the superreplication strategy of the contingent claim $f, \hat{x}$ being the initial value of this strategy. Note that the random variable 0 belongs to the set $\mathcal{D}(1,1)$, is anticomonotonic with $X_{T}+f$, and satisfies:

$$
\mathbb{E}\left(0\left(X_{T}-f\right)\right)=0=\hat{x}-\hat{x} .
$$

Applying Theorem (5) gives the corollary.

As Jouini and Kallal [14] already stressed, note that the superreplication strategies of the contingent claim $f$ are not necessarily replicating ones. Nevertheless, they always have a zero inefficiency size.

\subsection{Performance measure}

In this paragraph, we apply the results of the previous sections to the measure of performance. As in Dybvig [6] or Jouini and Kallal [14], we follow the tradition of comparing some investment strategy and its distribution of payoffs to the alternative of trading in a given securities market: the benchmark market. Since we do not assume that the market is frictionless, we have to take into account the uncertain future endowment because investment and hedging decisions can no longer be separated.

Given an uncertain future endowment $f$ in quantity $q_{0}$, an investment strategy is evaluated on the basis of the distribution $F_{c}$ of its net payoff $c$, where $c$ might depend on information not available to the agents (but only to the portfolio manager), allowing for information-trading and private investments outside the benchmark market. The benchmark market is described by the set $\mathcal{M}$ of supermartingales measures of pricing that summarize the investment opportunities that are available. For utility pricing, by Theorem (4), the relevant characteristic of the benchmark market is the set of cumulative distribution functions of the random variables belonging to $\bigcup_{p \in \mathcal{P}} \mathcal{D}(1, p)$. We then obtain the following result.

Suppose that an investment strategy leads from an initial wealth $\omega_{0}$ to a cumulative net distribution of payoffs $F_{c_{0}}$, with the hedging of a random endowment $f$ in quantity $q_{0}$ with $c \in L^{1}$. Let $V_{q_{0}, f}\left(c_{0}\right)=\sup _{p \in \mathcal{P}} \sup _{h \in \mathcal{D}(1, p)}\left\{\int_{0}^{1} F_{c}^{-1}(t) F_{h}^{-1}(1-\right.$ $t) d t-q_{0} p$. Then, 
1. if $\omega_{0}<V_{q_{0} f}\left(c_{0}\right)$, then there is superior performance, i.e. there exists a rational agent with concave and strictly increasing von NeumannMorgenstern preferences who prefers receiving the net distribution of payoffs $F_{c_{0}}$ to trading in the benchmark market. Moreover, the largest amount such that a rational agent would pay to switch is $V_{q_{0} f}\left(c_{0}\right)-$ $\omega_{0}>0$.

2. if $\omega_{0}=V_{q_{0}, f}\left(c_{0}\right)$, then there is ordinary performance, i.e., every rational agent with concave and strictly increasing von Neumann-Morgenstern preferences weakly prefers trading in the benchmark market to receiving the distribution of payoffs $F_{c_{0}}$. However, the lowest amount such that a rational agent would pay to switch is equal to zero.

3. If $\omega_{0}>V_{q_{0} f}\left(c_{0}\right)$, then there is inferior performance, i.e., every rational agent with concave and strictly increasing von Neumann-Morgenstern preferences strictly prefers trading in the benchmark market to receiving the distribution of payoffs $F_{c_{0}}$. Moreover, the lowest amount such that a rational agent would pay to switch is $\omega_{0}-V_{q_{0} f}(c)$.

Hence, by comparing the initial investment required by an investment strategy to the utility price of the distribution of its payoff we can evaluate its performance. If the utility price is lower than the initial investment, the portfolio is not well diversified and is underperforming. If the utility price is equal to the initial investment, the portfolio is well diversified and it is performing as it should. If the utility price is larger than the initial investment, the manager has superior ability and/or information and/or is subject to lower transaction costs, and the portfolio is overperforming.

This result gives an alternative to the Security Market Line (SML) in measuring portfolio performance. As opposed to the standard SML analysis, this alternative gives a correct evaluation even when a superior performance is due to private information. Indeed, the SML is based on mean-variance analysis, and even securities are assumed to be jointly normally distributed, they will typically not be normal once conditioned on information.

\section{A Appendix}

\section{Duality results and proof of Lemma (3.1)}

In this section, we prove duality results leading to Theorem (2) and Theorem (3.1). We first establish a bipolar relationship between the sets $\mathcal{C}(x, q)$ and $\mathcal{D}(y, r)$, giving us immediately Lemma (2). We then use a uniform integrability property in order to obtain the existence of a solution for the dual problem.

The following result, proved in the context of incomplete market by Hugonnier 
and Kramkov [10], is the necessary extension of the bipolar relationship between $\mathcal{C}(x)$ and $\mathcal{D}(y)$ to the case of expected utility maximization problem with an uncertain future random endowment at date $T$.

Proposition 3 1. Let $(x, q) \in \mathcal{K}$ and $c \geq 0$. The random variable $c$ belongs to $\mathcal{C}(x, q)$ if and only if:

$$
\mathbb{E}[h c] \leq x y+q r \text { for every }(y, r) \in \mathcal{G} \text { and } h \in \mathcal{D}(y, r) .
$$

2. Let $(y, r) \in \mathcal{G}$ and $h \geq 0$. The random variable $h$ belongs to $\mathcal{D}(y, r)$ if and only if:

$$
\mathbb{E}[h c] \leq x y+q r \text { for every }(x, q) \in \mathcal{K} \text { and } c \in \mathcal{C}(x, q) .
$$

Proof of Proposition 3..

We begin with a closure property of the sets $\mathcal{D}(y)$ and $\mathcal{D}(y, r)$. Then we prove respectively the first and second assertions of Proposition (3).

Let us first characterize the closure of $\mathcal{D}(y)$ and $\mathcal{D}(y, r)$. Let $(y, r) \in \mathcal{G}$. The sets $\mathcal{D}(y, r)$ and $\mathcal{D}(y)$ are closed for the topology of convergence in measure. We establish the result for $\mathcal{D}(y, r)$. Indeed, let $h_{n} \in \mathcal{D}(y, r)$ be a sequence which converges almost surely to a random variable $h$. First, we have by Fatou's Lemma, $\mathbb{E}\left(h\left(X_{T}+q f\right)\right) \leq x y+q r$ for every $X \in \mathcal{X}(x, q)$. By definition of $\mathcal{D}(y, r)$, there exists a sequence of supermartingales $Y^{n}$ belonging to $\mathcal{Y}(y, r)$ such that $Y_{T}^{n} \geq h_{n}$. Following Lemma 5.2 from Föllmer and Kramkov [8], there exists a sequence $\tilde{Y}^{n} \in$ $\operatorname{conv}\left\{Y^{n}, Y^{n+1}, \ldots\right\}, n \geq 1$, and a supermartingale with $Y_{0} \leq y$ such that $\tilde{Y}^{n}$ is Fatou-convergent to $Y$ on a dense countable set $\tau$ of $[0, T]$. Using Fatou Lemma, it is easy to see that $Y X$ is a supermartingale for each $X \in \mathcal{X}(x)$. Moreover, $Y_{T} \geq h$ and if we define:

$$
Z_{t}=\left\{\begin{array}{cl}
Y_{t}, & \text { if } t<T \\
h & t=T
\end{array} .\right.
$$

Then $Z \in \mathcal{Y}(y, r)$, and thus $h \in \mathcal{D}(y, r)$.

Let us now prove the first assertion. Suppose that $c \stackrel{a . s .}{\geq} 0$ and such that for every $(y, r) \in \mathcal{G}$ and $h \in \mathcal{D}(y, r)$, we have:

$$
\mathbb{E}[h c] \leq x y+q r \text { for some }(x, q) \in \mathcal{K}
$$

Let us prove that $c$ belongs to $\mathcal{C}(x, q)$. Let $p$ belong to $\tilde{P}$, and define the set $\mathcal{M}(p) \subset \mathcal{M}$ of measures $\mathbb{Q} \in \mathcal{M}$ such that $\mathbb{E}^{\mathbb{Q}}[f]=p$. We have obviously $\mathcal{M} \subset$ $Y(1)$. Furthermore, if $h_{T} \triangleq \frac{d \mathbb{Q}}{d \mathbb{P}}$, for some $\mathbb{Q} \in \mathcal{M}(p)$, and $X \in \mathcal{X}(x)$, we have:

$$
\mathbb{E}\left(h_{T}\left(X_{T}+q f\right)\right)=\mathbb{E}\left(h_{T} X_{T}\right)+q p \leq x+q p .
$$

Thus, $h_{T} \in \mathcal{D}(1, p)$ and:

$$
\mathbb{E}^{\mathbb{Q}}(c-q f) \leq x+q p-q p=x
$$

for every $\mathbb{Q} \in \mathcal{M}(p)$ and $p \in \mathcal{P}$. With the help of the superreplication Theorem (see Proposition 4.1 in Föllmer and Kramkov [8]), there exists a superreplication strategy of $c-q f$ in $\mathcal{X}(x)$, i.e. $c \in \mathcal{C}(x, q)$, and the first assertion is proved. 
We turn now to the second assertion. Let $h_{0} \geq 0,\left(y_{0}, r_{0}\right) \in \mathcal{G}$, and suppose that

$$
\mathbb{E}\left(h_{0} c\right) \leq x y_{0}+q r_{0}
$$

for every $(x, q) \in \mathcal{K}$ and $c \in \mathcal{C}(x, q)$. We want to prove that $h_{0} \in \mathcal{D}\left(y_{0}, r_{0}\right)$.

If $y_{0}=0$, since $r_{0} \leq 0$, necessarily $r_{0}=0$; Thus $h_{0}=0$, which belongs to $\mathcal{D}(0,0)$.

Now, let assume that $y_{0} \neq 0$. By the superreplication Theorem, the following property holds:

$$
c \in \mathcal{C}(1,0) \text { if and only if } \mathbb{E}(h c) \leq y_{0} \text { for every } h \in \mathcal{D}\left(y_{0}\right) .
$$

Thus, applying the bipolar Theorem (see Brannath and Schachermayer [1]), since $\mathcal{D}(y)$ is closed with respect to the topology of probability convergence, we have :

$$
h \in \mathcal{D}\left(y_{0}\right) \text { if and only if } \mathbb{E}(h c) \leq y_{0} \text { for every } c \in \mathcal{C}(1,0)
$$

which is the case for $h_{0}$ and thus $h_{0} \in \mathcal{D}\left(y_{0}\right)$. Therefore, there exists $Y \in \mathcal{Y}(y)$ such that $Y_{T} \stackrel{\text { a.s. }}{\geq} h_{0}$, and, in the same way as in the proof of the closure of $\mathcal{D}(y, r)$, we define a new process $Z$ by:

$$
Z_{t}=\left\{\begin{array}{cl}
Y_{t}, & \text { if } t<T \\
h_{0} & t=T
\end{array}\right.
$$

Then $Z$ belongs to $\mathcal{Y}(y, r)$ and thus, finally, $h_{0}$ belongs to $\mathcal{D}(y, r)$.

A straightforward application of this result is Theorem (2).

We shall need now the following result of uniform integrability. This property, already proved in Kramkov and Schachermayer [16] is a key result to obtain the existence of a solution to the dual problem.

Lemma A.1 Let $(y, r) \in \mathcal{G}$. The family of random variables $V(h)_{h \in \mathcal{D}(y, r)}^{-}$ is uniformly integrable.

We are now in a position to prove Lemma (3.1). We follow the sketch of the proof of Lemma (3.6) in Kramkov and Schachermayer [16]. We first restrict our study to the case of terminal wealth bounded by a positive integer $n$ in order to apply a minimax theorem. Then, using the uniform integrability of the family $V(h)_{h \in \mathcal{D}(y, r)}^{-}$for $(y, r) \in \mathcal{G}$, we let $n$ go to infinity and we prove the existence of the solution of the dual problem as well as the conjugate relationship between the functions $u$ and $v$.

Proof of Lemma (3.1).

We prove first that:

$$
v(y, r)=\sup _{(x, q) \in \mathcal{K}}[u(x, q)-x y-q r] \text { for }(y, r) \in \mathcal{G},
$$

and the fact that the optimal solution $\hat{h} \in \mathcal{D}(y, r)$ of $(2.4)$ exists as soon as $v(y, r)<$ $+\infty$.

First, we put aside the case $(y, r)=(0,0)$. Indeed, since only 0 belongs to $\mathcal{D}(0,0)$, we have $v(0,0)=\sup _{x>0} U(x)=\sup _{(x, q) \in \mathcal{K}} u(x, q)$ and the result is therefore trivial. Now, let $\left(y_{0}, r_{0}\right) \in \mathcal{G} \backslash\{(0,0)\}$. Consider the set $\mathcal{C}^{\perp}\left(y_{0}, r_{0}\right)$ defined as follows:

$$
\mathcal{C}^{\perp}\left(y_{0}, r_{0}\right) \triangleq\left\{c \in \mathcal{C}(x, q), \text { for }(x, q) \in \mathcal{K} \text { such that } x y_{0}+q r_{0} \leq 1\right\}
$$


We define, for $n>0$, the set $\mathcal{B}_{n}$ of $L^{\infty}$ by $\mathcal{B}_{n} \triangleq\{g|0 \leq| g \mid \leq n\}$. The set $\mathcal{B}_{n}$ is $\sigma\left(L^{\infty}, L^{1}\right)$-compact and $\mathcal{D}\left(y_{0}, r_{0}\right)$ is a closed-convex subset of $L^{1}$. Furthermore, the function $f_{n}$ defined on $\mathcal{D}\left(y_{0}, r_{0}\right) \times \mathcal{B}_{n}$ by:

$$
f_{n}(h, c) \triangleq \mathbb{E}[U(c)-c h]
$$

is obviously concave with respect to $c$, linear and continuous with respect to $h$ in the space $L^{1}$. One can therefore apply the minimax Theorem, stated in Strasser (Theorem 45.8 p. 239, in [19]), and we obtain:

$$
\sup _{c \in \mathcal{B}_{n}} \inf _{h \in \mathcal{D}\left(y_{0}, r_{0}\right)} f_{n}(h, c)=\inf _{h \in \mathcal{D}\left(y_{0}, r_{0}\right)} \sup _{c \in \mathcal{B}_{n}} f_{n}(h, c) .
$$

Now, let's study the convergence of both right and left sides of this equation, letting $n$ goes to infinity.

For the left side, one deduce first easily from the definition of $\mathcal{D}\left(y_{0}, r_{0}\right)$ and $\mathcal{C}^{\perp}\left(y_{0}, r_{0}\right)$ that $h \in \mathcal{D}\left(y_{0}, r_{0}\right)$ if and only if

$$
\mathbb{E}[h c] \leq 1 \text { for every } c \in \mathcal{C}^{\perp}\left(y_{0}, r_{0}\right) .
$$

Thus, from the bipolar Theorem (see Brannath and Schachermayer [1]), the random variable $c$ belongs to the closure $\tilde{\mathcal{C}}^{\perp}\left(y_{0}, r_{0}\right)$ of $\mathcal{C}^{\perp}\left(y_{0}, r_{0}\right)$ if and only if:

$$
\sup _{h \in \mathcal{D}\left(y_{0}, r_{0}\right)} \mathbb{E}(h c) \leq 1 .
$$

We deduce that:

$$
\sup _{c \in \mathcal{B}_{n}} \inf _{h \in \mathcal{D}^{\perp}\left(y_{0}, r_{0}\right)} \mathbb{E}[U(c)-c h]=\sup _{z>0} \sup _{c \in \mathcal{B}_{n} \cap z \tilde{\mathcal{C}}^{\perp}\left(y_{0}, r_{0}\right)} \mathbb{E}[U(c)-z] .
$$

Applying Lemma (11) from Hugonnier and Kramkov [10], we have:

$$
\sup _{c \in \mathcal{B}_{n} \cap z \tilde{\mathcal{C}}^{\perp}\left(y_{0}, r_{0}\right)} \inf _{h \in \mathcal{D}^{\perp}\left(y_{0}, r_{0}\right)} \mathbb{E}[U(c)-z]=\sup _{c \in \mathcal{B}_{n} \cap z \mathcal{C}^{\perp}\left(y_{0}, r_{0}\right)} \mathbb{E}[U(c)-z] .
$$

Thus:

$$
\begin{aligned}
\lim _{n \rightarrow+\infty} \sup _{c \in \mathcal{B}_{n}} \inf _{h \in \mathcal{D}^{\perp}\left(y_{0}, r_{0}\right)} \mathbb{E}[U(c)-c h] & =\sup _{z>0}\left\{\sup _{c \in \mathcal{C}^{\perp}\left(y_{0}, r_{0}\right)} \mathbb{E}(U(z c))-z\right\} \\
& =\sup _{(x, q) \in \mathcal{K}} u(x, q)-x y_{0}-q r_{0} .
\end{aligned}
$$

Now, for the right side of the equation, if we define $V_{n}(y)=\sup _{0<x \leq n} U(x)-x y$, we have:

$$
\inf _{h \in \mathcal{D}\left(y_{0}, r_{0}\right)} \sup _{c \in \mathcal{B}_{n}} \mathbb{E}[U(c)-c h]=\inf _{h \in \mathcal{D}\left(y_{0}, r_{0}\right)} \mathbb{E}\left[V_{n}(h)\right) \triangleq v^{n}\left(y_{0}, r_{0}\right) .
$$

Consequently, since we have, by the convergence of the left side of the equation (A.18):

$$
\lim _{n \rightarrow+\infty} v^{n}\left(y_{0}, r_{0}\right)=\sup _{(x, q) \in \mathcal{K}} u(x, q)-x y_{0}-q r_{0}
$$


it is sufficient to prove that $\lim _{n \rightarrow+\infty} v^{n}\left(y_{0}, r_{0}\right)=v\left(y_{0}, r_{0}\right)$. Obviously, we have $v^{n} \leq v$. Let $\left(h_{n}\right)_{n \geq 1}$ be a sequence in $\mathcal{D}\left(y_{0}, r_{0}\right)$ such that:

$$
\lim _{n \rightarrow+\infty} \mathbb{E}\left[V_{n}\left(h_{n}\right)\right]=\lim _{n \rightarrow+\infty} v^{n}\left(y_{0}, r_{0}\right) .
$$

Since the set $\mathcal{D}\left(y_{0}, r_{0}\right)$ is bounded in $L^{1}$, we can apply Komlòs Lemma (see Hall and Heyde [9]) and find a sequence $g_{n} \in \operatorname{conv}\left(h_{n}, h_{n+1}, \ldots\right), h_{n} \in \mathcal{D}\left(y_{0}, r_{0}\right)$, which converges almost surely to a random vector $\hat{h}$. The sequence $g_{n}$ and the limit $\hat{h}$ belong to $\mathcal{D}\left(y_{0}, r_{0}\right)$ by closure of $\mathcal{D}\left(y_{0}, r_{0}\right)$ with respect to the convergence in probability topology. Furthermore, from the convexity of $V_{n}$, we have:

$$
\mathbb{E}\left[V_{n}\left(g_{n}\right)\right] \leq \sup _{m \geq n} \mathbb{E}\left[V_{n}\left(h_{m}\right)\right] \leq \sup _{m \geq n} \mathbb{E}\left[V_{m}\left(h_{m}\right)\right]
$$

where the last inequality holds because of the increasing of the sequence $V_{n}($.$) .$ Moreover, the family $V_{n}\left(g_{n}\right)^{-}$is uniformly integrable. Indeed, we can choose $y_{0}>0$ such that there exists $x_{0}<1$ with $x_{0} \in \partial V\left(y_{0}\right)$. We have then $\partial V\left(y_{0}\right)=\partial V_{n}\left(y_{0}\right)=$ $\partial V_{1}\left(y_{0}\right)$ and $\partial V_{n}(y)=\partial V(y)$ as soon as $y>y_{0}$, i.e. $V_{n}(y)=V(y)$ as soon as $y>y_{0}$. We deduce then that:

$$
V_{n}\left(g_{n}\right)^{-} \leq V\left(g_{n}\right)^{-}+V\left(y_{0}\right)^{-} .
$$

The property of uniform integrability of the family $V_{n}\left(g_{n}\right)^{-}$comes then from the one of $\left(V\left(g_{n}\right)\right)^{-}$(see Lemma (A.1)).

This leads to the following inequalities, with Fatou's Lemma:

$$
\lim _{n \rightarrow+\infty} \mathbb{E}\left[V_{n}\left(h_{n}\right)\right] \geq \liminf _{n \rightarrow+\infty} \mathbb{E}\left[V_{n}\left(g_{n}\right)\right] \geq \mathbb{E}[V(\hat{h})] \geq v\left(y_{0}, r_{0}\right)
$$

which proves the equality (A.17), and the fact that if $v\left(y_{0}, r_{0}\right)<+\infty, \hat{h}$ is solution to the dual problem.

It only remains to prove that:

$$
u(x, q)=\inf _{(y, r) \in \mathcal{G}}[v(y, r)+x y+q r] \text { for }(x, q) \in \mathcal{K} .
$$

This equation is obtained by usual theory of conjugate functions, since $K$ is an open set belonging to the domain of $u$.

\section{A.1 Proof of Lemma (4.1).}

Proof of Lemma 4.1.

One can first remark that the following inequality is straightforward, for $c \in \mathcal{P}\left(c_{0}\right)$ :

$$
\inf _{c \in \mathcal{P}\left(c_{0}\right)} \sup _{p \in \tilde{\mathcal{P}}} \sup _{h \in \mathcal{D}(1, p)} \mathbb{E}(h c)-q_{0} p \geq \sup _{p \in \tilde{\mathcal{P}}} \sup _{h \in \mathcal{D}(1, p)} \inf _{c \in \mathcal{P}\left(c_{0}\right)} \mathbb{E}(h c)-q_{0} p
$$

Thus, we just need to prove the converse inequality in order to obtain the result. The sketch of the proof is as follows. We use the same scheme as in the proof of duality results for the utility maximization problem. We begin with a remark an then study bounded random variables with some integer $n$, finally letting $n$ go to infinity. 
Remark A.1 To verify that a terminal wealth $c \in L_{+}^{1}$ belongs to the set $\mathcal{P}\left(c_{0}\right)$, we could restrict our study to bounded and twice continuous differentiable utility functions. Indeed, one can always find a sequence of utility functions $U_{n} \in \mathcal{U} \cap$ $C_{b}^{2}\left(\mathbb{R}_{+}^{*}, \mathbb{R}\right)$, such that $\left|U_{n}(x)\right|$ is non decreasing and such that $\lim _{n \rightarrow+\infty} U_{n}(x)=$ $U(x)$. Then, the theorem of dominated convergence implies that if $\mathbb{E}\left(U_{n}(c)\right) \geq$ $\mathbb{E}\left(U_{n}\left(c_{0}\right)\right)$, then $\mathbb{E}(U(c)) \geq \mathbb{E}\left(U\left(c_{0}\right)\right)$.

With this remark, note that it is straightforward to see that $\mathcal{P}\left(c_{0}\right)$ is closed with respect to the topology of convergence in measure. For each $p \in \tilde{\mathcal{P}}$, we denote by $\mathcal{D}_{n}(1, p)$ the set $\mathcal{D}(1, p) \cap\{h \mid 0 \leq h \leq n\}$ The sets $\mathcal{D}_{n}(1, p)$ are weak convex compact subsets of $L^{1}$. Moreover with the theorem of dominated convergence, $(h, p) \rightarrow \mathbb{E}(h c)-q_{0} p$ is continuous. By the same minimax Theorem (Strasser [19]) as the one applied in the proof of Theorem (3), we obtain:

$$
\sup _{p \in \tilde{\mathcal{P}}} \sup _{h \in \mathcal{D}_{n}(1, p)} \inf _{c \in \mathcal{P}\left(c_{0}\right)} \mathbb{E}(h c)-q_{0} p=\inf _{c \in \mathcal{P}\left(c_{0}\right)} \sup _{p \in \tilde{\mathcal{P}}} \sup _{h \in \mathcal{D}_{n}(1, p)} \mathbb{E}(h c)-q_{0} p .
$$

Now, let $\mathrm{n}$ go to infinity and study the convergence of both sides of the equation (A.19). To do so, we define the function $\pi_{n}$ as

$$
\pi_{n}(c) \triangleq \sup _{p \in \tilde{\mathcal{P}}} \sup _{h \in \mathcal{D}_{n}(1, p)} \mathbb{E}(h c)-q_{0} p .
$$

The equation (A.19) implies in particular that:

$$
\begin{aligned}
\lim _{n \rightarrow+\infty} \inf _{c \in \mathcal{P}\left(c_{0}\right)} \pi_{n}(c) & =\lim _{n \rightarrow+\infty} \sup _{p \in \tilde{\mathcal{P}}} \sup _{h \in \mathcal{D}_{n}(1, p)} \inf _{c \in \mathcal{P}\left(c_{0}\right)} \mathbb{E}(h c)-q_{0} p \\
& \leq \sup _{p \in \tilde{\mathcal{P}}} \sup _{h \in \mathcal{D}_{n}(1, p)} \inf _{c \in \mathcal{P}\left(c_{0}\right)} \mathbb{E}(h c)-q_{0} p .
\end{aligned}
$$

Note that, for every random variable $c \in \mathcal{P}\left(c_{0}\right)$, we can always find a random variable $\tilde{c} \in \Sigma\left(c_{0}\right)$ with $\tilde{c} \leq c$ (Theorem (1)). Thus, we can choose a sequence $c_{n} \in \Sigma\left(c_{0}\right)$ such that

$$
\lim _{n \rightarrow+\infty} \inf _{c \in \mathcal{P}\left(c_{0}\right)} \pi_{n}(c)=\lim _{n \rightarrow+\infty} \pi_{n}\left(c_{n}\right) .
$$

Since $\mathbb{E}\left(c_{n}\right)=\mathbb{E}\left(c_{0}\right)$ (indeed $c_{n} \in \Sigma\left(c_{0}\right)$ ), one can apply the Komlos Theorem, and find a sequence $\tilde{c}_{n} \in \operatorname{conv}\left\{c_{n}, c_{n+1}, c_{n+2}, \ldots\right\}$ such that $\tilde{c}_{n} \rightarrow \tilde{c}$ almost surely. Since $\Sigma\left(c_{0}\right)$ is closed with respect to the topology of convergence in measure, the sequence $\tilde{c}_{n}$ and its limit $\tilde{c}$ belong to the set $\Sigma\left(c_{0}\right)$. We obtain:

$$
\begin{aligned}
\pi_{n}\left(\tilde{c}_{n}\right) & \leq \sup _{m \leq n} \pi_{n}\left(c_{m}\right) \\
& \leq \sup _{m \leq n} \pi_{m}\left(c_{m}\right)
\end{aligned}
$$

where the first inequality is obtained since $\pi_{n}$ is convex, and the second one by monotonicity of the sequence $\pi_{n}$. Moreover:

$$
\begin{aligned}
\pi_{n}\left(\tilde{c_{n}}\right) \geq \inf _{k \geq n} \pi_{n}\left(\tilde{c_{k}}\right) & \geq \sup _{p \in \tilde{\mathcal{P}}} \sup _{h \in \mathcal{D}_{n}(1, p)} \inf _{k \geq n} \mathbb{E}\left(h \tilde{c}_{k}\right)-q_{0} p \\
& \geq \sup _{p \in \tilde{\mathcal{P}}} \sup _{h \in \mathcal{D}_{n}(1, p)} \mathbb{E}\left(\inf _{k \geq n} h \tilde{c}_{k}\right)-q_{0} p=\pi_{n}\left(\inf _{k \geq n} h \tilde{c}_{k}\right)
\end{aligned}
$$


where the two first inequalities are straightforward and the third one is deduced from Fatou's Lemma. Finally, the theorem of monotone convergence gives:

$$
\begin{aligned}
\lim _{n \rightarrow+\infty} \pi_{n}\left(c_{n}\right) & \geq \lim _{n \rightarrow+\infty} \pi_{n}\left(\inf _{k \geq n} \tilde{c}_{k}\right) \\
& =\sup _{p \in \tilde{\mathcal{P}}} \sup _{h \in \mathcal{D}(1, p)} \mathbb{E}(h \tilde{c})-q_{0} p .
\end{aligned}
$$

Thus, using equation (A.20), we obtain:

$$
\begin{aligned}
\sup _{p \in \tilde{\mathcal{P}}} \sup _{h \in \mathcal{D}(1, p)} \inf _{c \in \mathcal{P}\left(c_{0}\right)} \mathbb{E}(h c)-q_{0} p & \geq \lim _{n \rightarrow+\infty} \pi_{n}\left(c_{n}\right) \\
& \geq \sup _{p \in \tilde{\mathcal{P}}} \sup _{h \in \mathcal{D}(1, p)} \mathbb{E}(h \tilde{c})-q_{0} p .
\end{aligned}
$$

As $\tilde{c} \in \mathcal{P}\left(c_{0}\right)$, we conclude that:

$$
\min _{c \in \mathcal{P}\left(c_{0}\right)} \sup _{p \in \tilde{\mathcal{P}}} \sup _{h \in \mathcal{D}(1, p)} \mathbb{E}(h c)-q_{0} p \leq \sup _{p \in \tilde{\mathcal{P}}} \sup _{h \in \mathcal{D}(1, p)} \inf _{c \in \mathcal{P}\left(c_{0}\right)} \mathbb{E}(h c)-q_{0} p .
$$

The other inequality is straightforward and the result is proved.

\section{References}

[1] W. Brannath and W. Schachermayer. A bipolar theorm for $L_{+}^{0}(\Omega, \mathcal{F}, \mathbb{P})$. Séminaire de probabilités, 1998.

[2] J. Cvitanic, W. Schachermayer, and H. Wang. Utility maximization in incomplete markets with random endowment. 2001.

[3] F. Delbaen and W. Schachermayer. The fundamental theorem of asset pricing for unbounded stochastic processes. Mathematische Annalen, 312:215-250, 1998.

[4] D. Denneberg. Non Additive Measure and Integral. Boston: Kluwer Academic Publishers, 1998.

[5] P.A. Diamond and J.E. Stiglitz. Increases in risk and in risk aversion. Journal of Economic Theory, 8(3), 1974.

[6] P.H. Dybvig. Distributional analysis of portfolio choice. Journal of Business, 63(3):369-393, 1988.

[7] P.H. Dybvig. Inefficient dynamic portfolio strategies or how to throw away a million dollars in the stock markets. Review of Financial Studies, 1(1):67-88, 1988.

[8] H. Föllmer and D. Kramkov. Optionnal decompositions under constraints. Probability Theory and Related Fields, 109:1-25, 1997. 
[9] P. Hall and C.C. Heyde. Martingale Limit Theory and its Application. Academic Press, New York.

[10] D. Hugonnier and D. Kramkov. Optimal investment with random endowments in incomplete markets. 14(2):845-864, 2004.

[11] E. Jouini and H. Kallal. Arbitrage in securities markets with short sales constraints. Mathematical Finance, 5(3):197-232, 1995.

[12] E. Jouini and H. Kallal. Martingales and arbitrage in securities markets with transactions costs. Journal of Economic Theory, 57(1):176-196, 1995.

[13] E. Jouini and H. Kallal. Viability and equilibrium in securities markets with frictions. Mathematical Finance, 9(3):275-292, 1999.

[14] E. Jouini and H. Kallal. Efficient trading strategies in the presence of market frictions. The review of Financial Studies, 14(2):343-369, 2001.

[15] I. Karatzas and S.E. Shreve. Methods of Mathematical Finance. Springer Verlag, 1998.

[16] D. Kramkov and W. Schachermayer. The asymptotic elasticity of utility functions and optimal investment in incomplet markets. Annals of Applied Probability, 9(3):904-950, 1999.

[17] M. Rothshild and J.E. Stiglitz. Increasing risk: I. a definition. Journal of Economic Theory, 2(3):225-243, 1970.

[18] M. Rothshild and J.E. Stiglitz. Increasing risk: Ii. its economic consequences. Journal of Economic Theory, 3(1):66-84, 1970.

[19] H. Strasser. Mathematical Theory of Statistics. de Gruyter Studies in Mathematics 7, 1985. 